\title{
Second-order theory for coupling 2D numerical and physical wave tanks: Derivation, evaluation and experimental validation
}

\author{
Yang, Zhiwen; Liu, Shuxue; Bingham, Harry B.; Li, Jinxuan
}

Published in:

Coastal Engineering

Link to article, DOI:

10.1016/j.coastaleng.2012.07.003

Publication date:

2013

Document Version

Publisher's PDF, also known as Version of record

Link back to DTU Orbit

Citation (APA):

Yang, Z., Liu, S., Bingham, H. B., \& Li, J. (2013). Second-order theory for coupling 2D numerical and physical wave tanks: Derivation, evaluation and experimental validation. Coastal Engineering, 71, 37-51.

https://doi.org/10.1016/j.coastaleng.2012.07.003

\section{General rights}

Copyright and moral rights for the publications made accessible in the public portal are retained by the authors and/or other copyright owners and it is a condition of accessing publications that users recognise and abide by the legal requirements associated with these rights.

- Users may download and print one copy of any publication from the public portal for the purpose of private study or research.

- You may not further distribute the material or use it for any profit-making activity or commercial gain

- You may freely distribute the URL identifying the publication in the public portal 


\title{
Second-order theory for coupling 2D numerical and physical wave tanks: Derivation, evaluation and experimental validation
}

\author{
Zhiwen Yang a,b,*, Shuxue Liu ${ }^{\text {a, }}{ }^{\text {, Harry B. Bingham }}{ }^{\text {b,2 }}$, Jinxuan Li ${ }^{\text {a }}$ \\ a State Key Laboratory of Coastal and Offshore Engineering, Dalian University of Technology, Dalian 116023, China \\ b Department of Mechanical Engineering, Technical University of Denmark, DK-2800 Kgs. Lyngby, Denmark
}

\section{A R T I C L E I N F O}

Article history:

Received 29 February 2012

Received in revised form 9 June 2012

Accepted 29 July 2012

Available online 30 August 2012

\section{Keywords:}

Second-order coupling

Numerical wave tanks

Physical wave tanks

Wavemaker theory

\begin{abstract}
A B S T R A C T
A full second-order theory for coupling numerical and physical wave tanks is presented. The ad hoc unified wave generation approach developed by Zhang et al. [Zhang, H., Schäffer, H.A., Jakobsen, K.P., 2007. Deterministic combination of numerical and physical coastal wave models. Coast. Eng. 54, 171-186] is extended to include the second-order dispersive correction. The new formulation is presented in a unified form that includes both progressive and evanescent modes and covers wavemaker configurations of the piston- and flap-type. The second order paddle stroke correction allows for improved nonlinear wave generation in the physical wave tank based on target numerical solutions. The performance and efficiency of the new model is first evaluated theoretically based on second order Stokes waves. Due to the complexity of the problem, the proposed method has been truncated at 2D and the treatment of regular waves, and the re-reflection control on the wave paddle is also not included. In order to validate the solution methodology further, a series of nonlinear, periodic waves based on stream function theory are generated in a physical wave tank using a piston-type wavemaker. These experiments show that the new second-order coupling theory provides an improvement in the quality of nonlinear wave generation when compared to existing techniques.

(c) 2012 Elsevier B.V. All rights reserved.
\end{abstract}

\section{Introduction}

Deterministic models have increasingly been used by coastal engineers for combining numerical and physical wave tanks. Indeed, this approach allows the numerical model to focus on wave propagation in the offshore region and the physical model to treat the wave transformation in the near-shore region. Wave generation in the physical model completely utilizes the calculated wave motions by a suitable numerical model along with the boundary data connecting the two models and considers the asymmetry and nonlinearity of the progressive wave. The data transfer between the two models is built upon real-time nonlinear wave motion instead of stochastic parameters such as significant wave height and peak period. Consequently, this approach exploits the advantages of numerical and physical modeling to provide an improved description of full-scale, realistic engineering problems.

Initial efforts in this direction include Zhang and Schäffer (2004) and Zhang and Schäffer (2007) for wave flumes, and Zhang and Schäffer (2005) for the theoretical aspects in 3D. The most advanced

\footnotetext{
* Corresponding author. Fax: + 4545884325.

E-mail addresses: ouyangzhiwen@gmail.com (Z. Yang), liusx@dlut.edu.cn (S. Liu), hbb@dtu.mek.dk (H.B. Bingham), lijx@dlut.edu.cn (J. Li).

${ }^{1}$ Fax: + 8641184708526 .

2 Fax: +4545884325
}

theory developed for this purpose is referred to as the ad hoc unified wave generation theory (Zhang et al., 2007), which blends linear, fully dispersive wavemaker theory with a nonlinear, shallow water wave generation approach. In this method, the wave paddle control signal is calculated from a nonlinear shallow wavemaker difference equation and then corrected with a linear dispersion parameter. This method has been experimentally verified to be well suited for generation of small amplitude waves in all water depths and for nonlinear shallow water waves. The method's performance deteriorates however for nonlinear waves in intermediate or deep water. In order to extend the range of application of this method, Yang et al. (2011b) proposed a real-time correction method to correct the error which is produced in the hydrodynamic frameworks and the mechanical system of wavemaker. However, while the dispersion correction or the real-time correction in previous studies are based on linear wavemaker theory, the evanescent modes are not accounted for in the paddle signals which renders them inadequate for correcting the high harmonic components which appear when generating highly nonlinear or relatively deep water waves. Quite recently Yang et al. (2011a) developed an approximate second-order coupling theory which pushes the limits of application from shallow water to relatively deep water, but this theory did not include the evanescent modes. Moreover, the previous models collectively ignored the unwanted spurious free waves in the physical tank. Therefore, in this paper we introduce a new second-order coupling theory for numerical and physical wave models which includes the evanescent modes. Relevant 
analyses regarding the generation and the corresponding suppression efficiency of the spurious free waves have also been addressed.

Since the accuracy of a coupling model mainly depends on the assumption of wave paddle motion and the description of the resultant physical wave field, we are motivated to extend the model to include second-order wavemaker theory. Second-order wavemaker theory has a long history of development going back at least to Fontanet (1961) for regular waves. Ottesen-Hansen et al. (1980), Sand (1982) and Sand and Donslund (1985) discussed the second-order long wave generation in the laboratory. Hudspeth and Sulisz (1991) derived the complete second-order Eulerian theory for waves generated by a monochromatic wave paddle motion with special emphasis on Stokes drift and return flow in wave flumes. In addition, Flick and Guza (1980) and Sulisz and Hudspeth (1993) addressed the effects of spurious super-harmonic waves. In seeking an explanation of these effects, Schäffer (1996) derived a complete second-order model including both sub- and super-harmonics. This full theory successfully predicts the second-order motion of the wave paddle required to produce a spatially homogeneous wave field at second order. In this study, we apply the complete second-order wavemaker theory given by Schäffer (1996), except that we only consider regular (monochromatic) waves. The extension to irregular waves is the subject of ongoing work.

In the present model, the first- and second-order solutions for the wave paddle position are obtained by combining wavemaker theory with a fully nonlinear, depth-integrated flux based on a numerical model. This development is based on a classical perturbation approach combined with Taylor expansion of the boundary conditions on the free surface, which is formulated in a unifying form that includes both progressive and evanescent mode and covers wavemaker configurations of the piston- and flap-type. Except being restricted by the inherent limitations of second-order Stokes wave theory, this theory does not assume the primary waves to be restricted by a narrow frequency band, shallow water, or small amplitude. With a secondorder dispersion correction and the consideration of evanescent modes in the control signal, this new formulation allows the physical model to capture more accurately the high order behavior of nonlinear wave motion.

The derivation and effectiveness of the new formulation have been evaluated numerically by considering a theoretical secondorder Stokes wave. To test the limits of the method, we attempt to generate a wide range of nonlinear waves based on stream function theory using a piston-type wavemaker and compare with the original model of Zhang and Schäffer (2007). For better comparison of the accuracy and efficiency of the present model with the previous method, no attempt is made to consider re-reflection control on the wave paddle, so that all the wavemaker signals are based solely on the calculated wave paddle position. These experiments show that the new model has a wider range of applicability than existing wave generation methods.

The remainder of this paper is structured as follows. In Section 2 the formulation of the second-order coupling theory are given. Section 3 describes a recipe for the generation of the complete control coupling signal including a general procedure of the connection between the numerical and physical model and the solution of the coupling difference equation. Section 4 presents a numerical analysis on the relevant first- and second-order coupling parameters. Section 5 evaluates the model in terms of the suppression of spurious second-order free waves and the accuracy and efficiency of the proposed numerical scheme for solving the coupling equations. Section 6 discusses the range of application. Experimental validation is provided in Section 7. Finally, conclusions are drawn in Section 8.

\section{Modeling the second-order coupling wave field}

\subsection{General description}

The geometry of the coupling model is illustrated in Fig. 1. The simulation area is divided into a numerical field $\Omega_{1}$ and a physical field $\Omega_{2}$. $\Omega_{1}$ represents the far wave field which allows numerical waves to be generated and propagate into the physical model, and $\Omega_{2}$ indicates the near-shore area with the complex physics where the physical waves are generated from a coupled wavemaker signal based on the numerical wave information. Any suitable theory or numerical wave model can be used in the far field $\Omega_{1}$. It is clear that the kernel of the coupling problem is the data transformation between the two models, i.e., how to obtain accurately the wave paddle signals from a given numerical wave in order that the physical waves can proceed successfully in $\Omega_{2}$.

In order to fully understand the complexity of the data transformation, the wavemaker has to be considered as a coupled system. In this context, it is important to appreciate the following. Applying a single-frequency, sinusoidal signal to the wave paddle in $\Omega_{2}$ will cause it to move and generate a wave field. This wave field induces hydrodynamic feedback, such as wave surface elevation or velocity, around the wave board. The nature of this hydrodynamic feedback is dependent on the full nonlinearity of the numerical waves at the boundary of the two fields and it is this nonlinear hydrodynamic feedback that determines, via the paddle controller, the position to be applied at the next time step. Zhang et al. (2007) has proposed a first-order coupling approach, namely the ad hoc unified wavemaker theory, coupling provided by the depth-averaged horizontal particle velocity based on a linear wave theory. In this paper, however, we extend this method to include the second-order dispersive correction by forcing the moving wave paddle to match the second-order boundary condition between the two models. Consequently, for each time step $t$, numerical and physical model must satisfy the matching condition at a specific location $x$, i.e.,

$U_{P}(x, t) \equiv U_{N}(x, t)$

where, subscripts " $N$ " and " $P$ " represent the numerical and physical solution, respectively, $U$ the depth-averaged horizontal particle velocity.

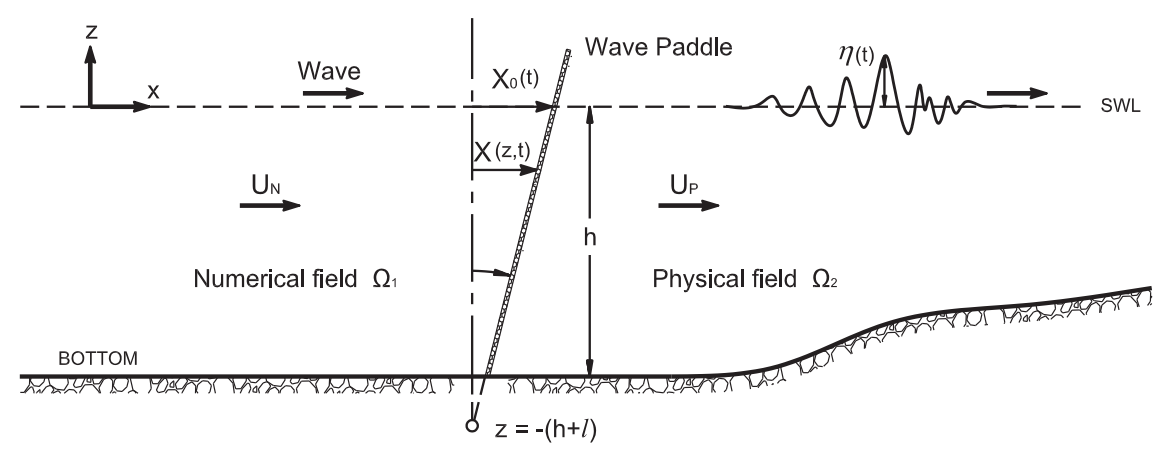

Fig. 1. Definition sketch of the coupled system of numerical and physical wave models. 


\subsection{Induced waves in physical field $\Omega_{2}$}

The second order calculation of wave field by giving a motion of the paddle has been extensively discussed see (e.g. Flick and Guza, 1980; Schäffer, 1996; Sulisz and Hudspeth, 1993). Throughout this paper the notation of Schäffer (1996) will be employed to describe the induced waves in the physical field $\Omega_{2}$. With the physical wave field known, the coupling relation between the numerical and physical model can be determined. Similar to Schäffer (1996), the induced wave potential solution can be separated into the following four components:

$\phi=\varepsilon \phi^{(1)}+\varepsilon^{2} \phi^{(2)}+O\left(\varepsilon^{3}\right)$

$\phi^{(2)}=\phi^{(21)}+\phi^{(22)}+\phi^{(23)}$

where $\varepsilon$ is a small ordering parameter proportional to the wave steepness $H / L$, in which $H$ is the wave height and $L$ the wavelength.

(1) The first-order velocity potential $-\phi^{(1)}$ : This velocity potential satisfies the first-order boundary condition on the free surface and the moving wave paddle, which describes the progressive wave to be generated as well as the associated evanescent modes arising at, or near, the wave paddle.

(2) The second-order bound wave velocity potential $-\phi^{(21)}$ : This gives the bound waves due to the cross-interaction between first-order wave components, which satisfies the second order free surface boundary condition. The bound waves are phase locked to the first order wave components.

(3) The second-order spurious free wave potential $-\phi^{(22)}$ : This potential describes the free waves due to the wavemaker leaving its mean position and due to the fact that $\phi^{(21)}$ mismatches the boundary condition at the wavemaker. If the control signal of the wavemaker only contains a first-order motion, then the resultant second-order surface elevation is given by $\phi^{(2)}=$ $\phi^{(21)}+\phi^{(22)}$, i.e., the spurious free waves expressed as $\phi^{(22)}$ will be generated.

(4) The additional second-order potential induced by second order numerical correction $-\phi^{(23)}$ : Although $\phi^{(21)}$ and $\phi^{(22)}$ together satisfy the second order free surface boundary condition, they do not fully satisfy the second-order boundary condition at the moving wave paddle. The additional potential $\phi^{(23)}$ is introduced, together with $\phi^{(21)}$ and $\phi^{(22)}$, to fully satisfy the second order boundary condition on the free surface and at the moving wave paddle, which generates a compensated wave field to eliminate the spurious free waves.

The solution of the first three velocity potential, $\phi^{(1)}, \phi^{(21)}$ and $\phi^{(22)}$, is identical to that presented by Schäffer (1996). In contrast, for the potential $\phi^{(23)}$ described in the pure second-order wavemaker theory, it can be obtained by counteracting the free wave potential, $\phi^{(22)}$, such that $\phi^{(23)}=-\phi^{(22)}$. However, for a second order coupling theory in the present paper, $\phi^{(23)}$ should be determined from a given second-order hydrodynamic feedback of depth-averaged horizontal particle velocity wave paddle motion in the numerical model. In order to determine the complete motion of the wave paddle, the coupled wave field has to be modeled.

\subsection{Coupling of numerical and physical wave field up to second order}

\subsubsection{First-order coupling solution}

The first-order coupling approach has been discussed by Zhang et al. (2007), but their analysis neglected the evanescent terms in the wavemaker signal. In the present paper, the first-order coupling solution therefore should be re-derived including the evanescent terms, and a complex representation is chosen.
Let the time series of the depth-averaged horizontal particle velocity and the wave paddle position be denoted by $U_{0}(t)$, and $X_{0}(t)$, respectively, in physical space, while $U_{a}(\omega)$, and $X_{a}(\omega)$ denote the equivalent complex Fourier amplitudes, i.e.,

$$
\begin{aligned}
& U_{0}(t) \stackrel{\text { Fourier transform }}{\Leftrightarrow} U_{a}(\omega) \\
& X_{0}(t) \stackrel{\text { Fourier transform }}{\Leftrightarrow} X_{a}(\omega)
\end{aligned}
$$

where subscripts " 0 " and " $a$ " represent the time and frequency domain respectively. The perturbation series of $(U, X)$ can be expanded as

$U=\varepsilon U^{(1)}+\varepsilon^{2} U^{(2)}+O\left(\varepsilon^{3}\right)$

$X=\varepsilon X^{(1)}+\varepsilon^{2} X^{(2)}+O\left(\varepsilon^{3}\right)$

In order to produce a coupling wave field, the first-order displacement of the wavemaker paddle needs to be firstly assumed to be

$X_{0}^{(1)}=\frac{1}{2}\left\{-i X_{a}^{(1)} e^{i \omega t}+\right.$ c.c. $\}$

where $X_{a}^{(1)}$ is the first-order complex paddle amplitude at the still water level, $\omega$ the angular frequency, and c.c. the complex conjugate of the preceding term. Then the solution to the first-order wave potential can be expressed as

$\phi^{(1)}=\frac{1}{2}\left\{\frac{i g X_{a}^{(1)}}{\omega} \sum_{j=0}^{\infty} c_{j} \frac{\cosh k_{j}(z+h)}{\cosh k_{j} h} e^{i\left(\omega t-k_{j} x\right)}+\right.$ c.c. $\}$

Here $\mathbf{i}$ is the imaginary unit showing a $90^{\circ}$ phase shift, the wave numbers, $k_{j}$, are the solutions to the first-order dispersion relation,

$\omega^{2}=g k_{j} \tanh k_{j} h$

This is the linear dispersion relation generalized to complex wave numbers, where $g$ denotes the acceleration of gravity. Eqs. (6) and (7) cover both the progressive-wave term $(j=0)$ and the evanescent modes $(j=1,2, \ldots)$. The coefficients $c_{j}$, are referred to as the Biésel transfer function, and are determined by requiring the solution to satisfy the boundary conditions at the wavemaker which are formulated in Appendix A. This paper will address two wavemaker configurations: piston-type $(l \rightarrow \infty)$ and flap-type $(l=0)$, see Fig. 1 .

According to the first-order velocity potential and applying the boundary condition Eq. (1), the relation between the numerical and physical models for the first-order depth-averaged horizontal particle velocities is given by

$U_{N}^{(1)}=U_{P}^{(1)}=\left.\frac{1}{h} \int_{-h}^{0} \phi_{x}^{(1)}\right|_{x=0} \mathrm{~d} z=\frac{1}{2}\left\{\omega X_{a}^{(1)} \sum_{j=0}^{\infty} \frac{c_{j}}{k_{j} h} e^{i \omega t}+\right.$ c.c. $\}$

Substituting Eq. (5) into Eq. (8) and considering the frequency domain expression, we get,

$\omega X_{a}^{(1)}(\omega)=\Lambda_{1 j}(\omega) \cdot U_{N, a}^{(1)}$

where $\Lambda_{1 j}$ is the first-order coupling function obtained by

$\Lambda_{1 j}(\omega)=\left[\sum_{j=0}^{\infty} \frac{c_{j}}{k_{j} h}+\text { c.c. }\right]^{-1}$ 
which includes both the progressive part and the evanescent modes. For $j=0$, i.e. without evanescent terms, Eq. (10) gives the real quantity $\Lambda_{10}$ which is known as the transfer function $\Lambda$ in Zhang et al. (2007). For $j=1,2, \ldots, \Lambda_{1 j}$ is purely imaginary.

Eq. (9) gives a frequency-domain relation between the wave paddle position for the physical model and the first-order component of the depth-averaged particle velocity exported from a numerical model. In practice, this is readily converted to the time domain. For convenient conversion, as in Zhang et al. (2007), it can firstly be rewritten as two equations

$\omega X_{a, s w}^{(1)}(\omega)=U_{N, a}^{(1)}$

$X_{a}^{(1)}(\omega)=\Lambda_{1 j}(\omega) \cdot X_{a, s w}^{(1)}(\omega)$

By means of the assumption of the first-order wave paddle motion, Eq. (5), the time-domain form can also be expressed as

$\frac{d X_{0, s w}^{(1)}(t)}{d t}=U_{N, 0}^{(1)}(t)$

$X_{0}^{(1)}(t)=F^{-1}\left[\Lambda_{1 j}(\omega) \cdot F\left[X_{0, s w}^{(1)}(t)\right]\right]$

where $F^{-1}$ and $F$ represent the inverse and forward Fourier transform, respectively. They are evaluated in practice via the Fast Fourier Transform (FFT). The subscript "sw" indicates the use of shallow water theory for obtaining the paddle position, since Eq. (12-a) is consistent with the idea which is widely applied in shallow long wave generation (see e.g. Synolakis, 1990). $U_{N, 0}^{(1)}$ denotes the time series of first-order depth-averaged particle velocity exported from a numerical model or a suitable wave theory. Eq. (12-a) formulates a real-time link between the numerical and physical models under the assumption of shallow water. Eq. (12-b) gives a first-order dispersion correction needed when deviating from the shallow water limit. Consequently, without the evanescent modes, Eqs. (12-a) and (12-b) are identical to the ad hoc unified wavemaker theory proposed by Zhang et al. (2007).

\subsubsection{Second-order coupling solution}

At second order, the idea will be consistent with the first order coupling solution, i.e., to seek the relation between the wave paddle position for the physical model and the depth-averaged particle velocity exported from a numerical model. The velocity potential $\phi^{(21)}$ and $\phi^{(22)}$ are identical to those of Schäffer (1996), which will be cited directly. With the present study limited to regular waves, only the superharmonics are important, i.e.,

$\phi^{(21)}=\frac{1}{2}\left\{\frac{i X_{a}^{(1)^{2}}}{2} \sum_{j=0}^{\infty} \sum_{l=0}^{\infty} \frac{H_{j l}^{+}}{D_{j l}^{+}} c_{j} c_{l} \frac{\cosh \left(k_{j}+k_{l}\right)(z+h)}{\cosh \left(k_{j}+k_{l}\right) h} e^{i\left(2 \omega t-k_{j} x-k_{l} x\right)}+c . c.\right\}$

and

$\phi^{(22)}=\frac{1}{2}\left\{\frac{i g c_{0}^{2} X_{a}^{(1)^{2}}}{2 h \omega} \sum_{p=0}^{\infty} c_{p}^{(22)+} \frac{\cosh K_{p}^{+}(z+h)}{\cosh K_{p}^{+} h} e^{i\left(2 \omega t-K_{p}^{+} x\right)}+\right.$ c.c. $\}$

with $H_{j l}^{+}, D_{j l}^{+}, K_{p}^{+}$and $c_{p}^{(22)+}$ given in Appendix A.

For the potential $\phi^{(23)}$ described in Schäffer (1996) in a pure second-order wavemaker theory, it can be obtained by counteracting the free wave potential, $\phi^{(22) \pm}$, such that $\phi^{(23) \pm}=-\phi^{(22) \pm}$. But, for a second-order coupling theory as in the present study, $\phi^{(23)}$, should be determined from the given second-order wave paddle motion by matching the second-order numerical wave field. This quantity, in fact, cannot be obtained directly since the second-order wave paddle motion is unknown. Therefore, analogous to the first-order problem, we firstly assume the second-order paddle motion as

$X_{0}^{(2)}=\frac{1}{2}\left\{-i X_{a}^{(2)} e^{2 i \omega t}+\right.$ c.c. $\}$

where $X_{a}^{(2)}$ denotes the second-order complex paddle amplitude at still water level, then $\phi^{(23)}$ will be obtained by

$\phi^{(23)}=\frac{1}{2}\left\{\frac{i g X_{a}^{(2)}}{2 \omega} \sum_{p=0}^{\infty} c_{p}^{(23)+} \frac{\cosh K_{p}^{+}(z+h)}{\cosh K_{p}^{+} h} e^{i\left(2 \omega t-K_{p}^{+} x\right)}+\right.$ c.c. $\}$

where $c_{p}^{(23)+}$ is given in Appendix A.

Similar to the separation of the velocity potential, the given second-order depth-averaged particle velocity in a numerical model can also be separated into the following three terms,

$U_{N}^{(2)}=U_{N}^{(21)}+U_{N}^{(22)}+U_{N}^{(23)}$

Making the depth-averaged integration of $\phi_{x}^{(2)}$ at $x=0$ in the same way as for $\phi_{x}^{(1)}$ in Eq. (8), and considering Eq. (4-a) and the boundary condition of Eq. (1), the relation between the second-order depthaveraged horizontal particle velocity in the numerical model and the second-order wave paddle position in physical models can be derived as

$U_{N}^{(21)}=\frac{1}{2}\left\{\frac{X_{a}^{(1)^{2}}}{2 h} \sum_{j=0}^{\infty} \sum_{l=0}^{\infty} \frac{H_{j l}^{+} c_{j} c_{l} \tanh \left(k_{j}+k_{l}\right) h}{D_{j l}^{+}} e^{2 i \omega t}+\right.$ c.c. $\}$

$U_{N}^{(22)}=\frac{1}{2}\left\{\frac{2 c_{0}^{2} \omega X_{a}^{(1)^{2}}}{h} \sum_{p=0}^{\infty} \frac{c_{p}^{(22)+}}{K_{p}^{+} h} e^{2 i \omega t}+\right.$ c.c. $\}$

$U_{N}^{(23)}=\frac{1}{2}\left\{2 \omega X_{a}^{(2)} \sum_{p=0}^{\infty} \frac{c_{p}^{(23)+}}{K_{p}^{+} h} e^{2 i \omega t}+\right.$ c.c. $\}$

Notice that once $X_{a}^{(1)}$ in first-order problem is known, $U_{N}^{(21)}$ and $U_{N}^{(22)}$ can be obtained. Applying the assumption of Eq. (15), $U_{N}^{(23)}$ can now be determined from

$U_{N}^{(23)}=\left\{2 i \omega X_{0}^{(2)} \sum_{p=0}^{\infty} \frac{c_{p}^{(23)+}}{K_{p}^{+} h}+c . c.\right\}$

Combining Eqs. (17-a), (17-b), and (17-c) with Eq. (18) gives

$2 \omega X_{a}^{(2)}(\omega)=\Lambda_{2 p}(\omega) \cdot\left[U_{N, a}^{(2)}-\nu_{12}(\omega)\right]$

where $\Lambda_{2 p}$ is the second-order coupling coefficient given by

$\Lambda_{2 p}(\omega)=\left[\sum_{p=0}^{\infty} \frac{c_{p}^{(23)+}}{K_{p}^{+} h}+\text { c.c. }\right]^{-1}$

As before, $\Lambda_{20}$ represents the real, and $\Lambda_{2 p}(p=1,2 \ldots)$ the purely imaginary. $v_{12}(\omega)$ is the complex cross-order depth-averaged particle velocity, found from

$\nu_{12}(\omega)=\lambda^{2}(\omega) X_{a}^{(1)} \cdot X_{a}^{(1)}$ 
with

$\lambda^{2}(\omega)=\left\{\frac{1}{2 h} \sum_{j=0}^{\infty} \sum_{l=0}^{\infty} \frac{H_{j l}^{+}}{D_{j l}^{+}} c_{j} c_{l} \tanh \left(k_{j}+k_{l}\right) h+\frac{2 c_{0}^{2} \omega}{h} \sum_{p=0}^{\infty} \frac{c_{p}^{(22)+}}{K_{p}^{+} h}+c . c.\right\}$.

Note that $\lambda^{2}(\omega)$ is the term in the cross-order coupling coefficient which depends on the relative water depth and contains the interaction between the first- and second-order problems.

Considering the solution in the frequency domain, as in the first-order problem, Eq. (19) can be split into two equations

$2 \omega X_{a, s w}^{(2)}(\omega)=U_{N, a}^{(2)}-v_{12}(\omega)$

$X_{a}^{(2)}(\omega)=\Lambda_{2 p}(\omega) \cdot X_{a, s w}^{(2)}(\omega)$

with the corresponding expressions in the time domain given by

$\frac{d X_{0, s w}^{(2)}(t)}{d t}=U_{N, 0}^{(2)}(t)-\nu_{12}(t)$

$X_{0}^{(2)}(t)=F^{-1}\left[\Lambda_{2 p}(\omega) \cdot F\left[X_{0, s w}^{(2)}(t)\right]\right]$

Here subscript "sw" again refers to the shallow water solution. Eq. (24-b) gives a second-order dispersion correction needed when deviating from the shallow water limit. The complete wave paddle motion in the physical model is now

$X_{0}(t)=X_{0}^{(1)}(t)+X_{0}^{(2)}(t)$.

The full second-order coupling theory between the numerical and physical models has now been derived. It represents a unifying and compact form that includes both progressive and evanescent mode contributions and includes wavemakers of the piston- and flap-type.

\section{Implementation}

Now that we have derived the coupling model up to second order, we will give the recipe for the generation of the complete coupling control signal. There are three aspects that have to be addressed. Whilst the first question involves the general procedure of the presented second-order coupling from a numerical model to a physical model, the second problem relates to the decomposition of the depth-averaged particle velocity and the last aspect deals with the solution of the coupling equation.

It is well known from the ad hoc wavemaker theory (Zhang et al., 2007; see also Yang et al., 2011b) that there are three steps to get a coupling control signal. Firstly, a suitable numerical model is used to simulate wave propagation from the far field to the boundary between the numerical and physical models. Then the time series of depth-averaged particle velocity at the connecting boundary must be determined, in which the first- and second-order components have to be calculated (from Eq. (26) to Eqs. (27-a) and (27-b)). Secondly, the first- and second-order components of control signal will be obtained to provide the total second-order signal in the time domain. Finally, the physical wave is generated using the complete wavemaker signal. The detailed description is given in Fig. 2 which provides a general procedure of the new control mode (double-headed and continuous lines). Fig. 2 also shows the first-order coupling control mode, i.e., the ad hoc unified wavemaker theory of Zhang et al. (2007) (single-headed and dotted line). It must be kept in mind that in the first-order coupling control mode, the control signal can be calculated directly from Eqs. (12-a) and (12-b) by taking the first-order component of depth-averaged velocity, $U^{(1)}$ to be the total velocity, $U$.

For calculating the wave paddle position, various mathematical tools could be employed to exactly decompose the total depthaveraged particle velocity $U$ calculated from the numerical model into its first- and second-order components, $U^{(1)}$ and $U^{(2)}$. In this paper, a frequency-spectrum Fourier analysis method is adopted. Converting the depth-average velocity from the time domain to the frequency domain, we get

$U(t)=\operatorname{Re} \sum_{j} \Delta_{j} e^{i\left(\omega_{j} t+\varphi_{j}\right)} \quad$ with $\quad e^{i \varphi_{j}} \Delta_{j}=\frac{1}{2 \pi} \int_{t} U(t) e^{-i \omega_{j} t} d t$

in which $\Delta_{j}$ and $\varphi_{j}$ correspond to the amplitude and phase component, respectively, of the depth-averaged particle velocity $U$ in the frequency-domain at radian frequency $\omega_{j}$. For the regular wave case the phase can be set as $\varphi_{j} \equiv 0$. Then the first- and second-order solutions in the time domain can be determined by taking the second- and first-order component in frequency domain to be 0 , respectively, and using an inverse Fourier transform, i.e.

$$
\begin{aligned}
& U^{(1)}(t)=F^{-1}\left[\left\{\begin{array}{ll}
\Delta_{1}, & \omega=\omega_{1} \\
0, & \omega=\omega_{2} \\
\cdots & \text { others }
\end{array}\right]\right. \\
& U^{(2)}(t)=F^{-1}\left[\left\{\begin{array}{ll}
0, & \omega=\omega_{1} \\
\Delta_{2}, & \omega=\omega_{2} \\
0, & \text { others }
\end{array}\right] .\right.
\end{aligned}
$$

Finally, we consider the solution of the coupling equation. The depth averaged velocity $U(t)$ in Eqs. (12-a) and (24-a) are modified accordingly as $U\left(X_{0, s w}(t), t\right)$ in order to include the effect of the wave paddle position to yield

$\frac{d X_{0, s w}^{(r)}(t)}{d t}=U^{(r)}\left(X_{0, s w}^{(r)}(t), t\right)$

Note that Eq. (28) is a nonlinear equation with " $r$ " representing either first- or second-order. Since the numerical model may not have many grid points distributed over the range of the moving paddle, the nonlinear term $U\left(X_{0, s w}(t), t\right)$, was interpolated by Zhang (2005) using a 'spline' method to smooth out the distribution of the velocity near the moving paddle. They also used the explicit forward Euler (1st order) scheme for the discretization of time derivative terms. Furthermore, in order to avoid a slow drift of the paddle due to the discrepancy between the exact signals and the numerical

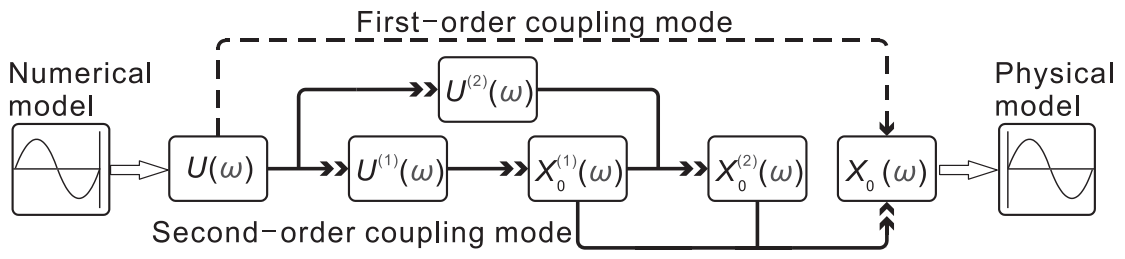

Fig. 2. General procedure of the coupling control modes. 
integration, a small term proportional to the paddle signal $\omega_{c} X_{0, s w}(t)$ ( $\omega_{c}$ the characteristic angular frequency) is added to the left hand side of the differential equation. However, the choice of the characteristic angular frequency $\omega_{c}$, is arbitrary and may lead to noticeable calculated errors.

In this paper, we adopt a new numerical scheme (see Section 5.2 for a detailed discussion regarding the accuracy and efficiency). The numerical solution for the depth-averaged horizontal particle velocities $U$ are saved at five fixed locations surrounding the mean paddle position, with $\Delta x$ being the spatial step-size between each of the five locations, and interpolation is used to get the instantaneous values. For the time-integration, the classical fourth-order RungeKutta scheme is used:

$X^{i}=X^{i-1}+\frac{\Delta t}{6}\left(\psi_{1}+2 \psi_{2}+2 \psi_{3}+\psi_{4}\right)$.

Here, the superscript "sw" and subscript " 0 " have been omitted for simplicity; $\Delta t$ is time step size, and $\psi_{1}, \psi_{2}, \psi_{3}$ and $\psi_{4}$ are the interpolated coefficients determined from

$\psi_{1}=\Gamma_{i-1}\left(X^{i-1}\right)$

$\psi_{2}=\frac{1}{2}\left[\Gamma_{i-1}\left(X^{i-1}+\Delta t \cdot \psi_{1} / 2\right)+\Gamma_{i}\left(X^{i-1}+\Delta t \cdot \psi_{1} / 2\right)\right]$

$\psi_{3}=\frac{1}{2}\left[\Gamma_{i-1}\left(X^{i-1}+\Delta t \cdot \psi_{2} / 2\right)+\Gamma_{i}\left(X^{i-1}+\Delta t \cdot \psi_{2} / 2\right)\right]$

$\psi_{4}=\Gamma_{i}\left(X^{i-1}+\Delta t \cdot \psi_{3}\right)$.

These terms are obtained using Lagrange interpolation between the five stored values of the velocity $U$ :

$\Gamma_{i}(\xi)=\sum_{j=1}^{5} u_{j}^{i} \cdot l_{j}[\xi]$

where $u_{j}^{i}$ represents the $U$ values exported from the numerical model at time step $i$, position $j$, and $l_{j}[\xi]$ is the interpolation function

$l_{j}[\xi]=\frac{\left(\xi-x_{1}\right) \cdots\left(\xi-x_{j-1}\right)\left(\xi-x_{j+1}\right) \cdots\left(\xi-x_{5}\right)}{\left(x_{j}-x_{1}\right) \cdots\left(x_{j}-x_{j-1}\right)\left(x_{j}-x_{j+1}\right) \cdots\left(x_{j}-x_{5}\right)}, \quad(j=1,2 \ldots 5)$.

\section{Coupling coefficients and their relevant analyses}

Before presenting the results, the correctness of the computer code is verified numerically by reproducing some numerical results described by Hudspeth and Sulisz (1991) and Sulisz and Hudspeth (1993). Fig. 3 shows a comparison of the ratio of the amplitudes of the second-order, far-field, free wave $\left(A^{(22)}\right.$ arising from $\left.\phi^{(22)}\right)$ to the bound wave $\left(A^{(21)}\right.$ arising from $\left.\phi^{(21)}\right)$ with the two-dimensional solution of Sulisz and Hudspeth (1993). Two curves are given; one corresponds to the piston-type wavemaker and the other the flaptype. In both cases, only the progressive wave part is considered. It is apparent that excellent agreement is observed over the entire frequency range of interest.

Having verified the accuracy of the associated computer code for the limiting case of monochromatic waves, some numerical results will now be presented for the first- and the second-order theory. All of the comparisons are plotted against relative water depths and for the two wavemaker configurations.

For the first-order coupling coefficient, Eq. (10) includes both real $(j=0)$ and imaginary $(j=1,2 \ldots)$ parts, which are shown in Figs. 4 and 5 , respectively. This complementary imaginary expression can

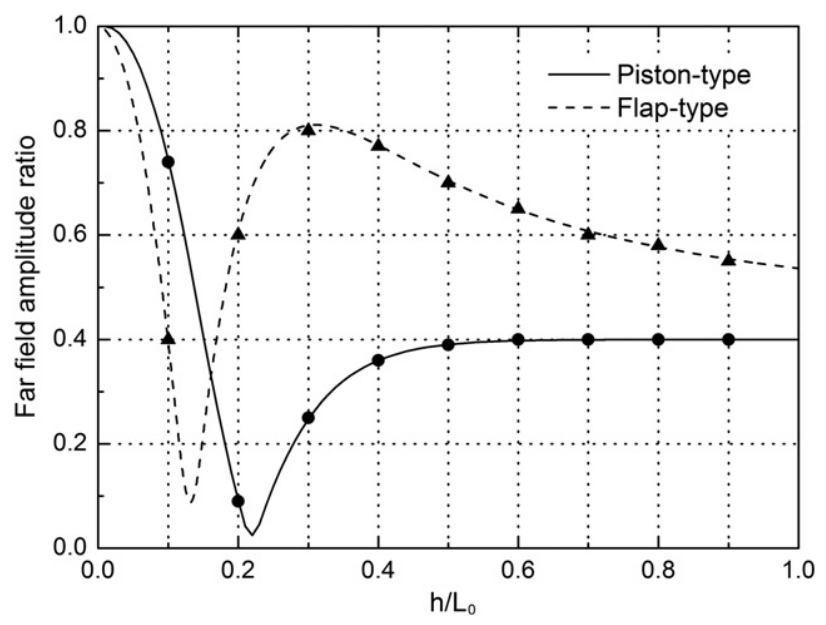

Fig. 3. Ratio of free second-order wavemaker and Stokes wave amplitude in far field for piston-type $(l \rightarrow \infty)$ and flap-type $(l=0)$ wavemaker. Comparison of results from the present method [lines] with that of Sulisz and Hudspeth (1993) [symbols] for monochromatic wavemaker motions.

be regarded as an extension of those given by Zhang et al. (2007). As noted in Zhang (2005), the contribution of the evanescent modes is insignificant in shallow water, which led them to only include the progressive part. The new components, however, do change the results of the wavemaker signal significantly in some specific conditions, evidence of which can be found in Fig. 6. For example, in case of the piston-type wavemaker, the contribution of evanescent components is tiny and can be neglected when $h / L_{0}<0.15$. This is a relatively shallow water case and the progressive part is hence significant. However, with the relative water depth increasing this proportion is substantial, contributing maximally about $25.1 \%$ to the total value when $h / L_{0}=0.63$. Examining further the contribution greater than or equal to $20 \%$, we can get the corresponding range of relative water depth $h / L_{0}=0.42-1.15$. For a flap-type of wavemaker the equivalent peak value occurs at $h / L_{0}=0.39$, range $h / L_{0}=0.26-0.68$. Indeed, these ranges correspond to relatively deep water cases for most coastal wave modeling and thus can reasonably be neglected in relatively shallow water studies.

For the second-order coupling coefficient, the form of Eq. (20) is consistent with the first-order coupling except for the second-order wave number $K_{p}^{+}$. Figs. 7 and 8 similarly plot the real $(p=0)$ and

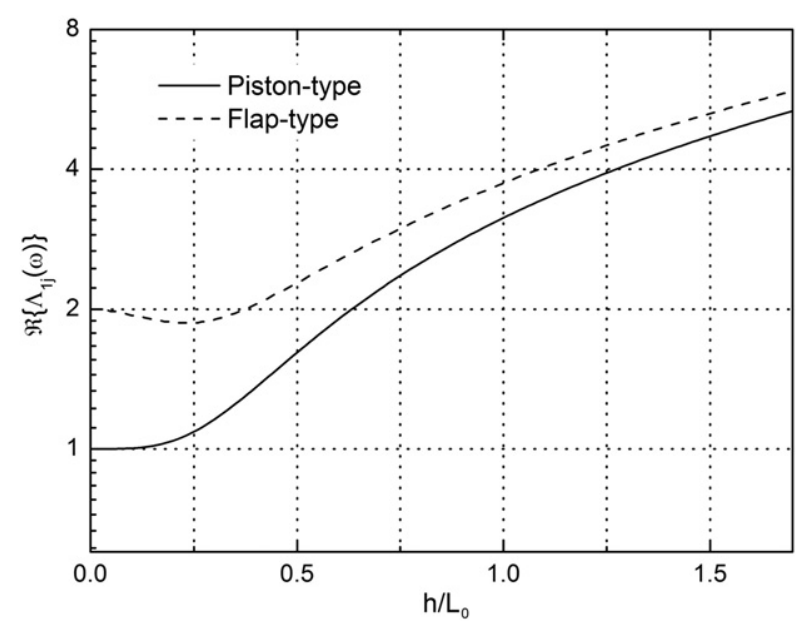

Fig. 4. The real part of the first-order coupling coefficients versus relative water depth for piston- and flap-type wavemakers. 


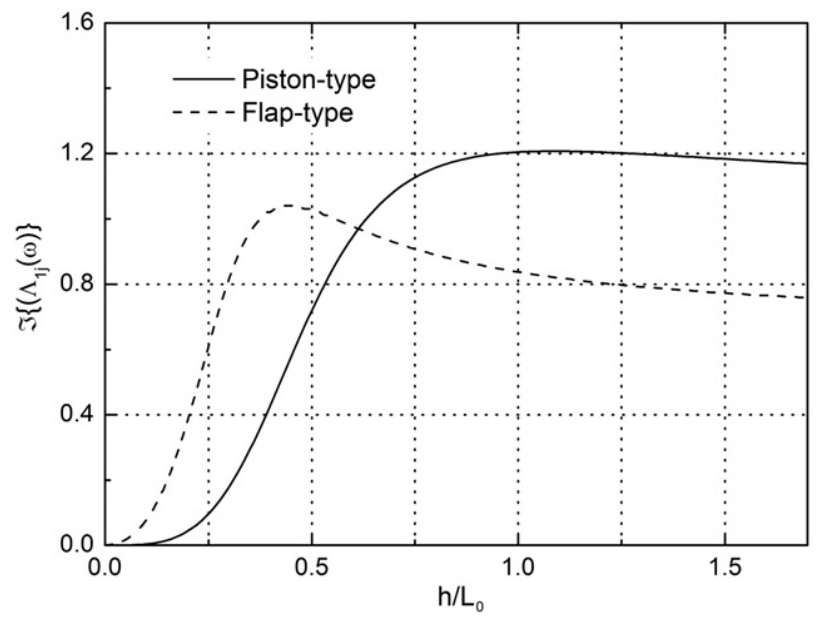

Fig. 5. The imaginary part of the first-order coupling coefficients versus relative water depth for piston- and flap-type wavemakers

imaginary $(p=1,2 \ldots)$ part of the second-order coupling coefficients versus relative water depth. From this plot, together with Figs. 4 and 5, we can see that the second-order coupling coefficient exhibits a similar trend to the first-order coefficients. Fig. 9 shows the contribution of the evanescent components to the total value for the second-order terms. For a piston-type wavemaker, we see that the maximum contributed proportion by the evanescent terms is $25.1 \%$ and the corresponding relative water depth is $h / L_{0}=0.37$. The region where the relative contribution is $\geq 20 \%$ occurs for $h / L_{0}=0.29-0.52$. For the flap-type wavemaker, the corresponding values are $h / L_{0}=$ 0.28 for the maximum point and the range $h / L_{0}=0.22-0.38$.

It is important to emphasize that for extremely shallow water conditions with the relative water depth approaching 0 , we get

$$
\left\{\begin{array}{l}
\Lambda_{2} \rightarrow \Lambda_{1} \\
\lambda^{2} \rightarrow 0
\end{array} \quad \text { for } h / L_{0} \rightarrow 0 .\right.
$$

Thus the present second-order coupling theory tends towards the first-order method of Zhang et al. (2007) in this limit. The common asymptotic value gives different results depending on the type of wavemaker. For example from Figs. 4 and 7, the limit is 1 for a piston type wavemaker and 2 for a flap-type. Furthermore, neglecting the

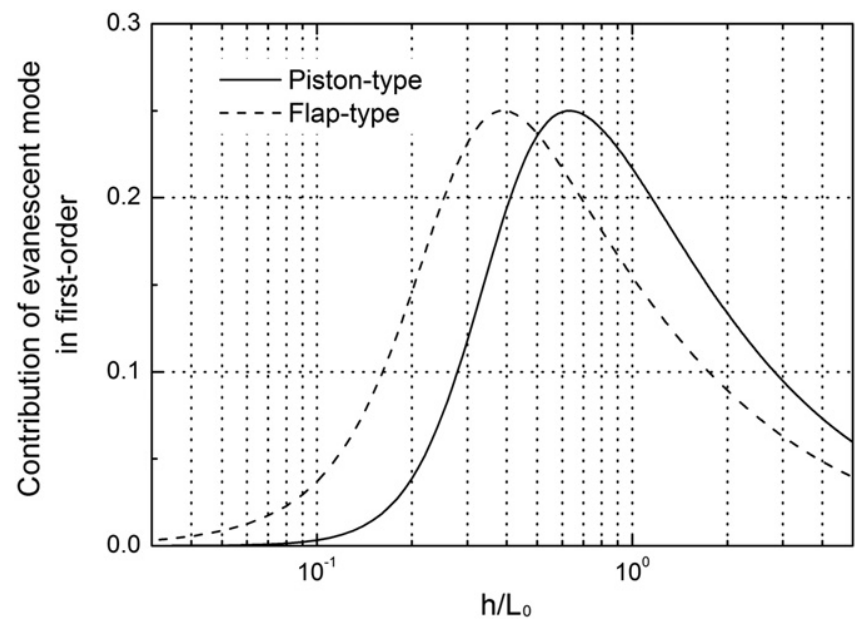

Fig. 6. Contribution of the evanescent modes in the first-order coupling coefficients for piston- and flap-type wavemakers.

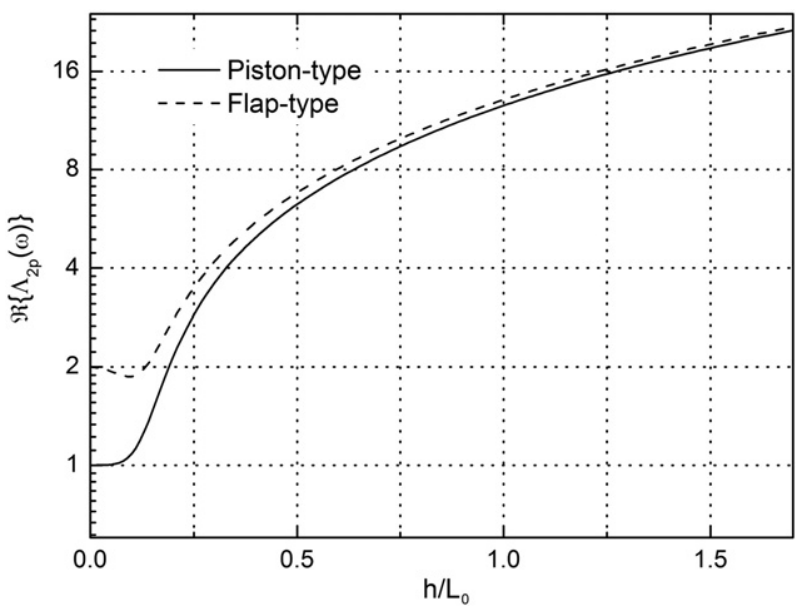

Fig. 7. The real part of the second-order coupling coefficients versus relative water depth for piston- and flap-type wavemakers.

evanescent modes the current theory also makes it collapse to the approximate second-order coupling theory derived by Yang et al. (2011a), regardless of whether it is in shallow or deep water.

Figs. 10 and 11 present the real and imaginary parts of the cross-order coupling coefficient for the two wavemaker configurations. It can be observed from these two figures that the cross-order coupling coefficient grows continuously with increasing relative water depth and has the shallow water limit of 0 . This is to demonstrate that the nonlinear interaction between first- and second-order is negligible in relatively shallow water and considerable in relatively deep water.

\section{Model evaluations}

\subsection{Suppression of the spurious free waves}

By analogy with a pure second-order wavemaker theory, a qualified second-order coupling signal should be calculated avoiding the existence of unwanted spurious second order free waves in the physical model domain. To this end, we present here several expressions and numerical results for evaluating the performance of the proposed second-order coupling theory. These will be illustrated by the evaluations for the suppression of the second-order spurious free waves.

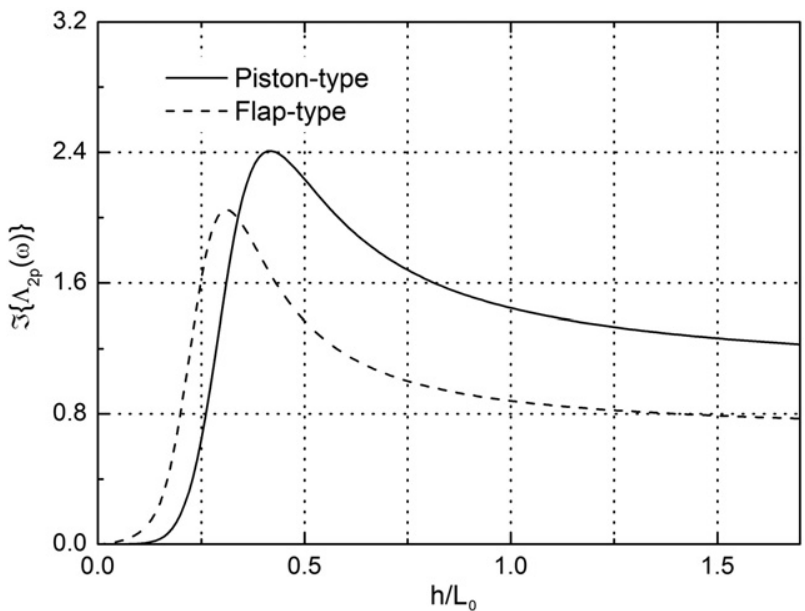

Fig. 8. The imaginary part of the second-order coupling coefficients versus relative water depth for piston- and flap-type wavemakers. 


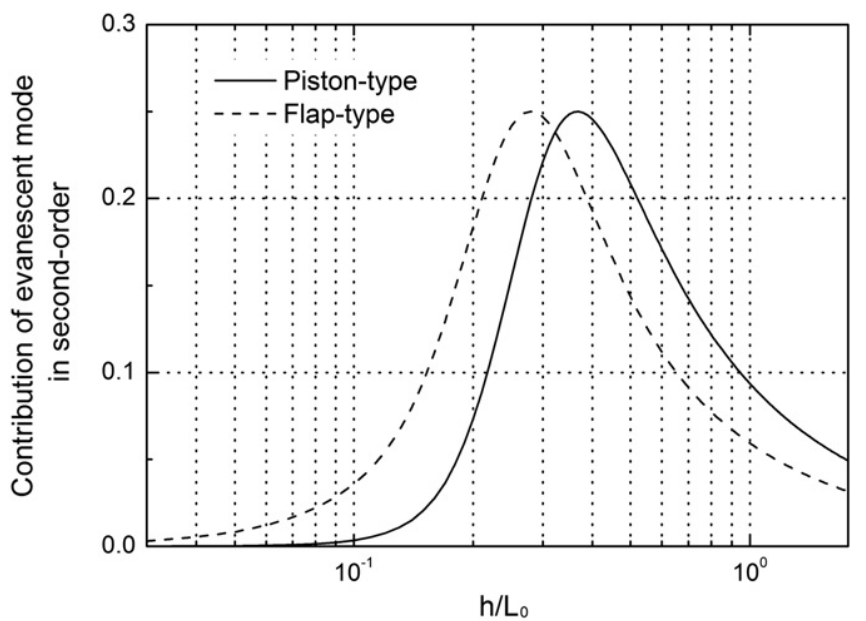

Fig. 9. Contribution of the evanescent modes in the second-order coupling coefficients for piston- and flap-type wavemakers.

A theoretical second-order Stokes wave is invoked as a test wave to evaluate the behavior.

Before estimating the efficiency of the proposed coupling model on the suppression of spurious second-order free waves, we should first introduce the theoretical solution for wave generation without spurious free waves in a physical model. Madsen (1971) pointed out, in a pure second-order wavemaker theory, that the spurious second-order free wave that is produced by a sinusoidally moving wave paddle could be theoretically eliminated by specifying the second-order wave paddle motion $X_{0}^{(2)}$ such that $\partial \phi_{22} / \partial x=0$. Here $\phi_{22}$ corresponds to the sum of $\phi^{(22)}$ and $\phi^{(23)}$ of Schäffer (1996). In terms of this assumption, the second-order paddle amplitude can be obtained by

$X_{a}^{(2)}=\frac{F^{+} c_{0}^{2}}{h} X_{a}^{(1)^{2}}$

where the transfer function $F^{+}$is found by ensuring that the progressive part of the spurious free wave and the progressive part of the additionally generated wave cancel out. The definition of $F^{+}$is given in Appendix A.

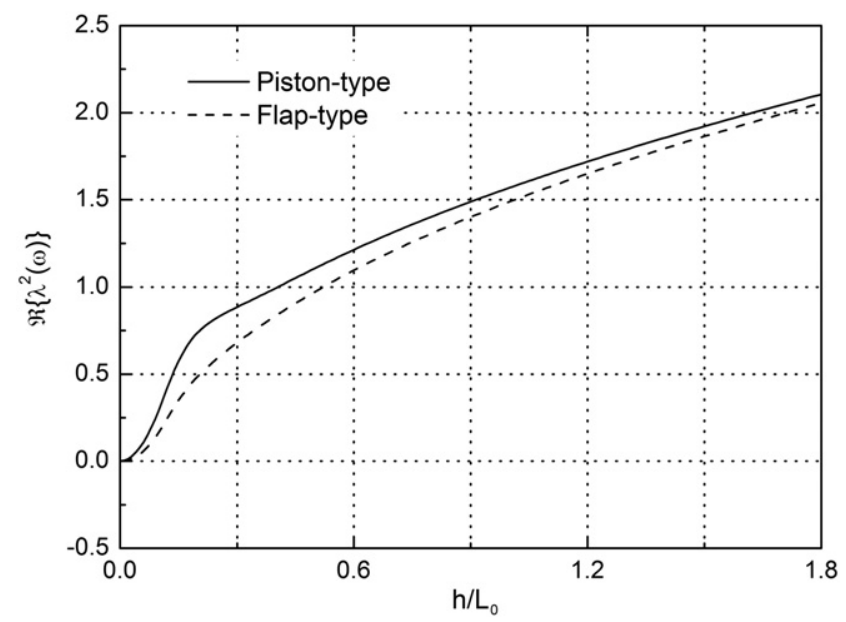

Fig. 10. The real part of the cross-order coupling coefficients versus relative water depth for piston- and flap-type wavemakers.

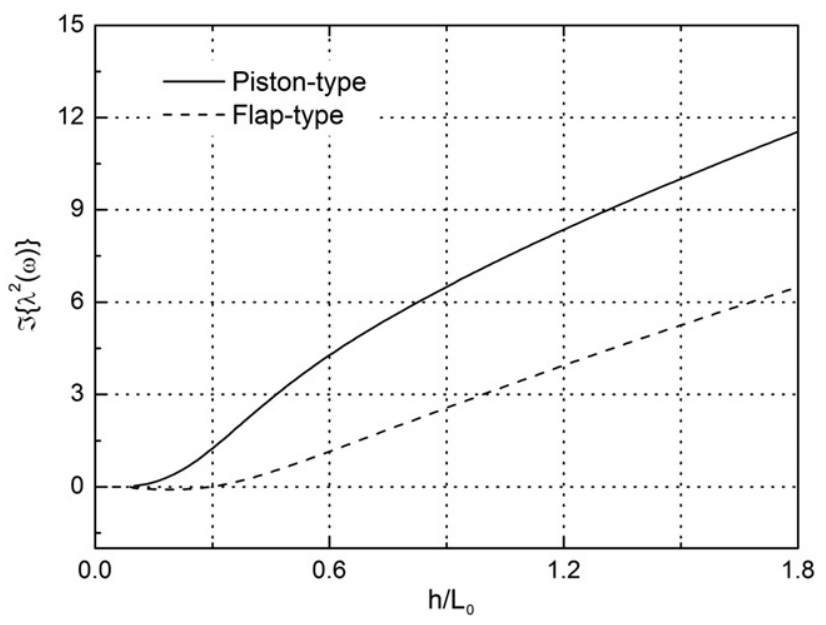

Fig. 11. The imaginary part of the cross-order coupling coefficients versus relative water depth for piston- and flap-type wavemakers.

It is more convenient to use the normalized form of second-order paddle amplitude of Eq. (34) to define the ideal suppression strength of spurious free waves. According to the first-order free surface condition, and neglecting evanescent modes yields: $X_{\mathrm{a}}^{(1)} c_{0}=H$ (see Schäffer, 1996), Eq. (34) can be re-written as

$\left.\frac{X_{a}^{(2)}}{X_{a}^{(1)}}\right|_{\text {suppress }}=R_{t}^{(2 / 1)}(\omega) \frac{H}{h}$.

Eq. (35) is defined as the theoretical relative second-order compensation of the paddle amplitude. It is obvious that the calculated relative second-order amplitude from the coupling model deviating from this quantity will lead to spurious second-order free waves. $R_{t}^{(2 / 1)}(\omega)$ denotes the theoretical disturbance parameter, where the superscript "(2/1)" means the ratio of the second-order to the firstorder and subscript " $t$ " the theoretical value, given by

$R_{t}^{(2 / 1)}(\omega)=F^{+} c_{0}$

$R_{\mathrm{t}}^{(2 / 1)}(\omega)$ obviously depends on the relative water depth $h / L_{0}$ since both $F^{+}$and $c_{0}$ are functions of $h / L_{0}$. It is hence deduced from Eq. (35)
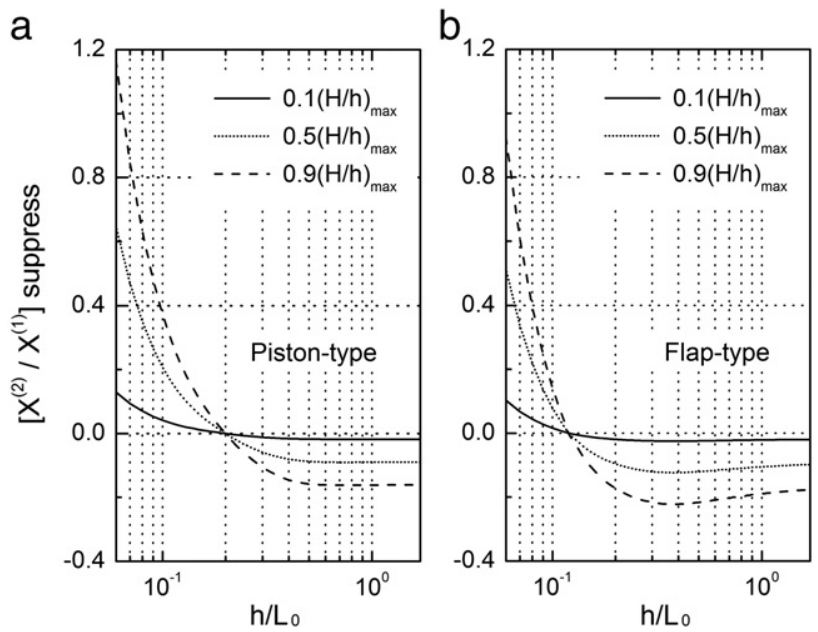

Fig. 12. Theoretical relative second-order compensation of paddle amplitude required for (a) the piston-type wavemaker and (b) the flap-type wavemaker, where three wave cases are considered; 0.1, 0.5, 0.9 times of the limiting relative wave height 
that this quantity varies only with the relative water depth $h / L_{0}$ (dispersion) and the relative wave height $H / h$ (nonlinearity), diagrams of which are provided in Fig. 12. Two wavemaker configurations and three wave cases $(H / h) /(H / h)_{\max }=0.1,0.5$ and 0.9 are plotted, where $(H / h)_{\max }$ is the limiting wave height found from Williams (1981), see also Fenton, 1990) for a stable, periodic wave. Two points are notable from this plot. First, in rather shallow water a larger second-order compensation of the wave paddle motion is required while in deep water a smaller value is required. Second, in both types of wavemaker, the theoretical relative second-order compensation of paddle amplitude grows with increasing relative wave height. Of particular interest is that the value 0 occurs at $h / L_{0}=0.21$ in piston-type wavemaker and $h / L_{0}=$ 0.12 in flap-type wavemaker.

To compare the discrepancy between the theoretical relative second-order compensation of wave paddle amplitude, Eq. (35), and the calculated value, we now consider the actual relative second-order paddle amplitude obtained from the coupling model using a theoretical second-order Stokes wave. These solutions are formulated in the same unified form as the theoretical solution. Again, two wavemaker configurations are included. Comparison of the current model (hereinafter referred as to "second-order coupling model") is also made to the ad hoc unified wave generation theory of Zhang et al. (2007) (hereinafter referred as to "First-order coupling model").

Neglecting the evanescent terms in second-order Stokes wave potential, the depth-averaged velocity of the progressive wave can be obtained by applying the second-order velocity potential (Dean and Dalrymple, 1991), that is,

$U=U^{(1)}+U^{(2)}=\frac{H \omega}{2 k h} e^{i \omega t}+\frac{3 H^{2} \omega \cosh k h}{16 h \sinh ^{3} k h} e^{2 i \omega t}$

with $H$ being the given wave height. Inserting Eq. (37) into the second- and the first-order coupling model, respectively, the wave paddle position can be obtained. It should be emphasized that the calculated wave paddle signal in first-order coupling model must contain second order components, since there is an inherent second order component included in the input depth-averaged velocity of Eq. (37). For the first-order coupling mode, the relative secondorder paddle amplitude can be obtained as

$\left.\frac{X_{a}^{(2)}}{X_{a}^{(1)}}\right|_{\text {First }}=R_{1}^{(2 / 1)}(\omega) \frac{H}{h}$

$R_{1}^{(2 / 1)}(\omega)=\frac{3 k h \cosh k h}{8 \sinh ^{3} k h}$

and, for second-order coupling control model, the equivalent solution is expressed as

$\left.\frac{X_{a}^{(2)}}{X_{a}^{(1)}}\right|_{\text {Second }}=R_{2}^{(2 / 1)}(\omega) \frac{H}{h}$

$R_{2}^{(2 / 1)}(\omega)=\frac{\Lambda_{2}}{\Lambda_{1}}\left(\frac{3 k h \cosh k h}{16 \sinh ^{3} k h}-\frac{k h^{2} \lambda^{2}}{4 c_{0}^{2} \omega}\right)$.

Similarly, the subscripts " 1 " and " 2 " in $R^{(2 / 1)}(\omega)$ describe the disturbance parameters arising from the second-order coupling mode and the first-order coupling mode, respectively.

As previously discussed in the theoretical solution, the relative second-order paddle amplitude obtained from the second-order coupling or the first-order coupling model depends on the relative water depth $h / L_{0}$ and the relative wave height $H / h$. Leaving aside the effect of $H / h$, Fig. 13 compares the disturbance parameters found from the second-order coupling model with the first-order coupling model for the two wavemaker configurations. In both models, the curve is compared to the theoretical solution. The curve obtained from the a

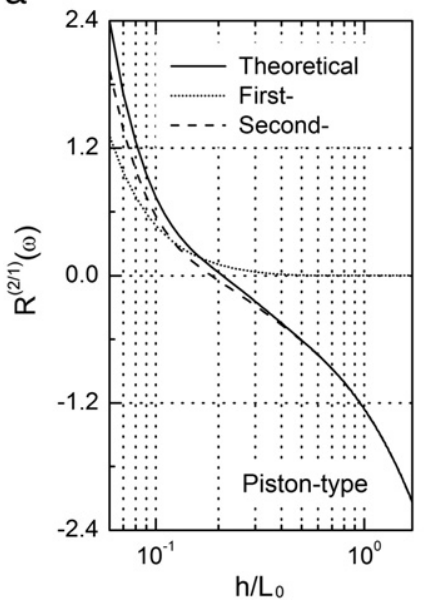

b

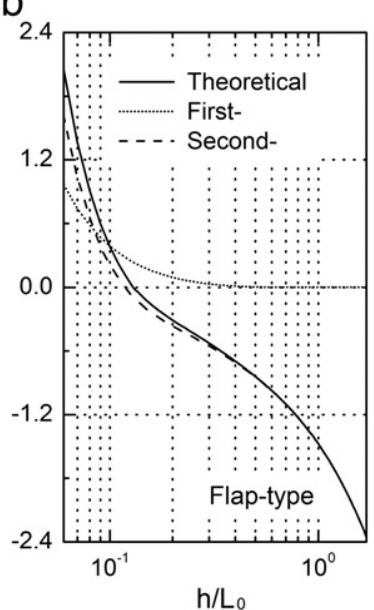

Fig. 13. Comparison of the disturbance parameters found from second-order coupling mode with first-order coupling mode for (a) the piston-type wavemaker and (b) the flap-type wavemaker.

second-order coupling model has a tendency which follows the theoretical curve at larger relative water depths. This is especially true for $h / L_{0}>0.5$ where the second-order model tends perfectly to the theoretical curve. At smaller relative water depth, corresponding to shallow water, the deviations between theoretical and second-order coupling solutions are apparent. This is mainly due to the fact that the validity of Stokes second-order wave hypotheses in Eq. (37) gradually vanishes as relative depth $\left(h / L_{0}\right)$ decreases (see e.g. Dean and Dalrymple, 1991). In the coupling model, the total spurious wave is given by the sum of the original spurious wave and the wave that is used to eliminate the original content by the coupling-induced paddle motion at second order, $X^{(2)}$. In the shallow water wave region, the inherent invalidity of Stokes second-order hypotheses is hence significant, so that the calculated relative second-order paddle amplitude obtained from the second-order coupling model tends to have difficulty matching the desired solution. Nevertheless, for most of the region of interest, the second-order coupling solution is much better than a first-order coupling.

Finally, we consider the practical development of the spurious second-order free waves. Fig. 14 compares the ratio of the total second-order, spurious free waves to the Stokes bound waves, $\left(A^{(22)}+A^{(23)}\right) / A^{(21)}$, found from the second-order coupling model
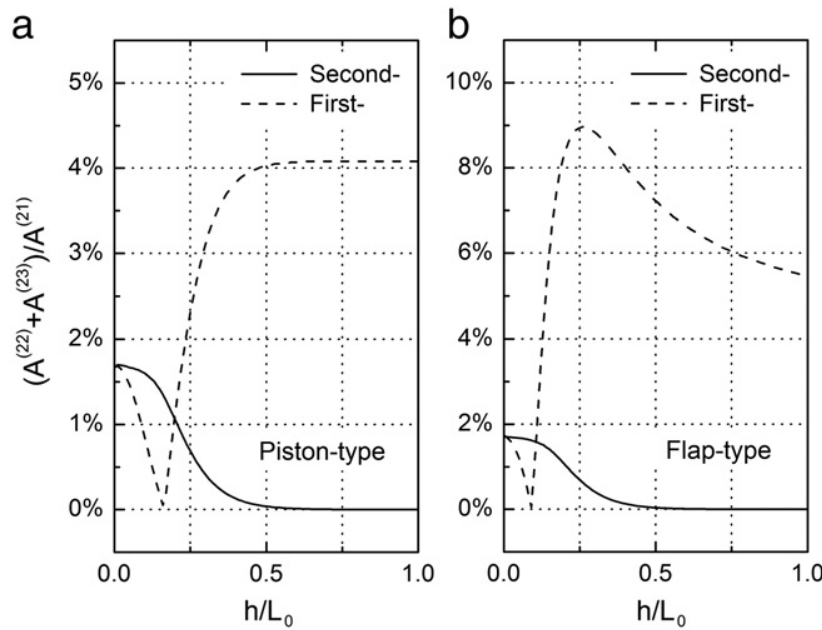

Fig. 14. Comparison of relative spurious second-order free waves due to the second-order coupling mode with the first-order coupling mode for (a) the piston-type wavemaker and (b) the flap-type wavemaker. 
with the first-order coupling model, where (a) is for the piston-type wavemaker and (b) for the flap-type. This plot illustrates two general features. First, the second-order coupling model clearly cancels out a significant proportion of the original spurious second order free wave of the first-order coupling method. For example, in the flap-type wavemaker, the maximum values of the dimensionless ratio, $\left(A^{(22)}+A^{(23)}\right) / A^{(21)}$, occurring at $h / L_{0}=0.25$ in the first-order coupling model is reduced by roughly $90 \%$ through second-order coupling. Of particular interest is that, in deep water $h / L_{0}>0.5$, the spurious wave content is completely eliminated when using the second-order coupling model. Second, it is only towards relatively shallow water that the difference between the two approaches reduces. In practice, however, this effect is hardly noticeable since the actual resultant spurious wave content is, on one hand, very limited, only around $1.6 \%$ when $h / L_{0} \rightarrow 0$, and, on the other hand, in shallow water the spurious wave wavenumber $K_{p}$ (found from $(2 \omega)^{2}=\mathrm{g} K_{p} \tanh K_{p} h$ ) tends to the bound wave wavenumber $2 k$ (found from $\omega^{2}=g k \tanh k h$ ). This means that the spurious free waves become the same harmonic as the primary bound waves, leading to a both spatially and temporally homogeneous wave field again. Similar results can also be seen for the piston-type wavemaker, the only difference being that the critical value 0 in the first-order coupling occurs at $h / L_{0}=0.14$. Furthermore, the piston-type wavemaker is found to be better for suppressing the spurious second-order free waves than the flap-type wavemaker in a coupling wave model.

\subsection{Accuracy and efficiency of the numerical scheme}

At the end of Section 3, we have proposed a new numerical scheme for the solution of the nonlinear coupling equations, Eq. (28), which needs to be verified. To quantify the accuracy and efficiency of the numerical scheme discussed above, we consider here a linear periodic wave characterized by height $H$, a period $T$ and the water depth $h$. It is well-known that the linearized wave problem has a theoretical solution, i.e., $U=\operatorname{Re}\left\{(\omega H / 2 k h) e^{\mathbf{i}(k x-\omega t)}\right\}$ and $X_{\text {lin. }}=\operatorname{Re}\left\{\left(-\mathbf{i} H / 2 c_{0}\right) e^{\mathbf{i}(k x-\omega t)}\right\}$, where $X_{\text {lin. }}$ denotes the wave paddle position obtained in linear wavemaker theory. As an example, we use Eqs. (29)-(32) to discretize the first-order coupling term in Eqs. (12-a) and (12-b) by taking $U^{(1)}$ to be $U$. The tests were run for a total of five periods after which the relative error per period was computed from

Error $=\frac{\left\|X_{5}-X_{\text {lin. }}\right\|_{2}}{5\left\|X_{\text {lin. }}\right\|_{2}}, \quad\|\cdot\|_{2}$ =two norm

where $X_{5}$ is the computed wave paddle position after exactly five wave periods. As a comparison, the method of Zhang (2005) has also been used to solve the same coupling equation. Fig. 15(a) collects the errors for the present numerical scheme on the first-order coupling equation as functions of the time and spatial resolutions $N_{t}$ and $\Delta x$, respectively, where we have taken $N_{t}=T / \Delta t$ so that $N_{t}$ represents the number of grid points per wave period. A linear wave characterized by $(T, H, h)=$ $(1.5 \mathrm{~s}, 0.05 \mathrm{~m}, 0.5 \mathrm{~m})$ was used for all tests, corresponding to $\left(h / L_{0}\right.$, $\left.H / L_{0}\right)=(0.18,0.018)$. For each case, the time resolutions $N_{t}$ are $[8,10$, $20,30,50,100,500]$ and the spatial resolution $\Delta x$ are $[1,2,3,5,7,10] \times$ $10^{-2} \mathrm{~m}$. This plot makes it clear that all the errors increase with increasing spatial step $\Delta x$ and decreasing number grid points per wave period $N_{t}$. Fig. 15(b) shows the comparison of error on the present numerical scheme with those of Zhang (2005). The maximum spatial step $\Delta x=0.1 \mathrm{~m}$ chosen in Fig. 15(a), and the characteristic angular frequency (normalized by the angular frequency of the target wave) $\omega_{c} / \omega$ of $[0.1,0.4,0.7,1.0,1.2]$ were used. As can be seen in the scheme of Zhang (2005), the numerical error depends on the choice of the normalized characteristic frequency $\omega_{c} / \omega$. It is also clear that, a significant improvement in accuracy can be obtained using the present numerical scheme. This is not surprising since the present scheme is formally fourth-order accurate in both spatial- and time-differencing, while the
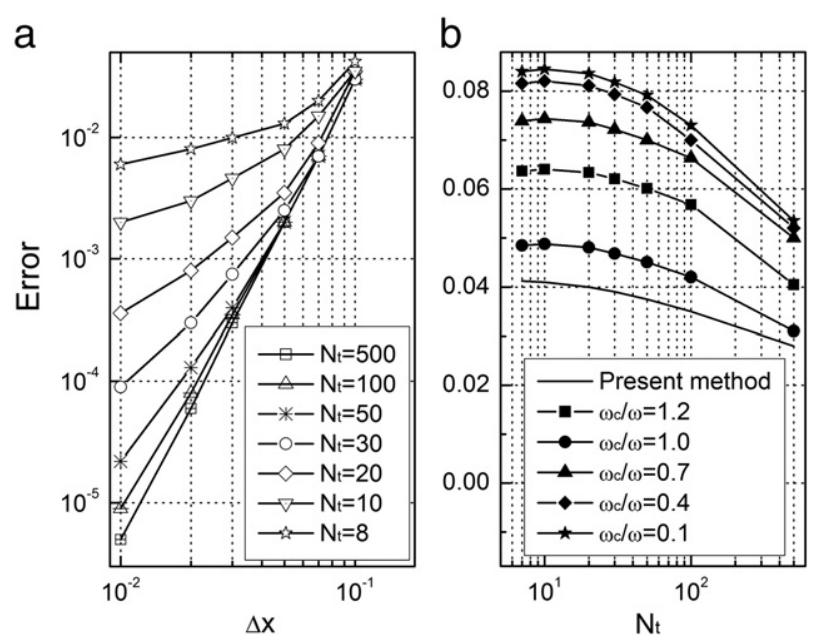

Fig. 15. Relative error Eq. (40) of the numerical scheme for solving the coupling equation; (a) for the present numerical scheme, $\Delta x$ values are at $[1,2,3,5,7,10] \times 10^{-2} \mathrm{~m}$; and (b) a comparison between the present method and the one of Zhang (2005) (lines with markers), $N_{t}$ points values are $[8,10,20,30,50,100,500]$.

methods used in Zhang (2005) are only first- and second-order. The proposed numerical scheme is generally satisfactory.

\section{Range of application}

In shallow water conditions, it is well known that the Stokes theory over predicts the second-order component even for very moderate wave steepness. Schäffer (1996) introduced a nonlinearity parameter $S$; a value of $S=1$ corresponding to the limiting case when a secondary peak appears in the trough of the primary wave. Consequently, for $S>1$ the theory fails to generate the correct second-order content. For a regular wave case, the nonlinearity parameter $S$ is given by

$$
S=2 H\left|\frac{\omega H^{+}}{2 g^{2} k \tanh 2 k h-4 g \omega^{2}}-\frac{1}{4 g}\left(\frac{g^{2} k^{2}}{\omega^{2}}-3 \omega^{2}\right)\right|
$$

where $\mathrm{H}^{+}$follows from $\mathrm{H}_{j k}^{+}$but only considers the progressive wave component

$H^{+}=3 \omega^{3}-\frac{3 g^{2} k^{2}}{\omega}$

Waves violating this criterion can be generated using a numerical technique such as Flick and Guza (1980), or perhaps in some instances waves might be better represented by Stream function wave theory.

\section{Experimental validation}

To further verify the proposed model, we now present experimental results for several test cases of theoretical wave generation. Since the stream function theory of Fenton (1988) has been successfully used by Zhang and Schäffer (2007) we consider this theory here to test the second-order coupling theory.

The depth-averaged horizontal particle velocity $U(x, t)$ can be expressed by means of continuity equations (Dean and Dalrymple, 1991)

$U(x, t)=\frac{c \eta(x, t)}{h+\eta(x, t)}$ 
where the surface elevation $\eta(x, t)$ is calculated in the Stream Function theory, which is expanded in Fourier functions as

$\eta(x, t)=\sum_{j=1}^{N} A_{j} \cos [j k(x-c t)]$

in which, $k$ is the first-order wave number, $c$ the phase velocity, and $A_{1 \ldots N}$ are constants for a given wave height $H$, water depth $h$ and period T.

The experiments were carried out in the wave flume of the Technical University of Denmark. The flume measures $0.75 \mathrm{~m}$ wide, $0.80 \mathrm{~m}$ deep, and $23 \mathrm{~m}$ long, and is equipped with a piston-type wavemaker driven by an electric drive system including a brushless AC motor and an integrated linear drive/bearing system at one end $(x=0)$. At the far end, a passive absorbing beach is installed. The wavemaker is controlled by the DHI AWACS system in position mode, where the control signal is a time series of wavemaker paddle position, which is the traditional method for nonlinear wave generation. In order to compare the accuracy of the present model with the existing method, no attempt is made to consider re-reflection control on the wave paddle, however, all measurements are completed before any reflected wave energy can return to the measurement locations from the beach.

The sample frequency of the coupling wavemaker signal is fixed at $0.02 \mathrm{~s}$. For each wave case, two control signals, the first-order coupling signal and the second-order coupling signal, are used. Three wave gauges at $1.0 \mathrm{~m}$ (gauge 1), $4.5 \mathrm{~m}$ (gauge 2), and $6.5 \mathrm{~m}$ (gauge 3) from the mean paddle position are fixed in the flume to measure the time series of surface elevation. The wave phases are shifted for each recorded signal to get a wave crest at $t=0$ for better comparison of wave profiles at each wave gauge location. Minor offsets from zero mean elevation were removed from the signals. All the cases (see Table 1) are fixed with water depth $h=0.5 \mathrm{~m}$. Various wave periods and heights are chosen for considering the dimensionless water depth $k h$ and nonlinearity $H / L_{0}$, where $L_{0}$ is the wavelength in deep water according to linear theory. At each $k h$, five values of wave height $H$ corresponding to $H / H \_m a x=0.1,0.3,0.5,0.7$ and 0.9 are given, where H_max is found from Williams (1981) (see also Fenton, 1990) and

Table 1

Wave cases tested in the flume with $h=0.5 \mathrm{~m}$.

\begin{tabular}{|c|c|c|c|c|c|}
\hline Case & $\begin{array}{l}\text { Wave period, } \\
T \text { (s) }\end{array}$ & $\begin{array}{l}\text { Wave height, } \\
H(\mathrm{~m})\end{array}$ & $\begin{array}{l}\text { Dimensionless } \\
\text { water depth, } k h\end{array}$ & $\begin{array}{l}\text { Wave } \\
\text { steepness, } \\
H / L_{0}\end{array}$ & $\begin{array}{l}\text { Nonlinearity } \\
\text { parameter, } S\end{array}$ \\
\hline 1 & 2.40 & 0.32 & 0.63 & 0.064 & 3.13 \\
\hline 2 & & 0.25 & & 0.050 & 1.45 \\
\hline 3 & & 0.18 & & 0.036 & 0.94 \\
\hline 4 & & 0.11 & & 0.022 & 0.78 \\
\hline 5 & & 0.08 & & 0.016 & 0.56 \\
\hline 6 & 1.60 & 0.28 & 1.04 & 0.093 & 1.12 \\
\hline 7 & & 0.22 & & 0.073 & 1.03 \\
\hline 8 & & 0.16 & & 0.053 & 0.87 \\
\hline 9 & & 0.09 & & 0.030 & 0.54 \\
\hline 10 & & 0.05 & & 0.017 & 0.30 \\
\hline 11 & 1.20 & 0.23 & 1.53 & 0.112 & 0.97 \\
\hline 12 & & 0.18 & & 0.088 & 0.79 \\
\hline 13 & & 0.13 & & 0.064 & 0.57 \\
\hline 14 & & 0.08 & & 0.039 & 0.35 \\
\hline 15 & & 0.04 & & 0.020 & 0.18 \\
\hline 16 & 1.00 & 0.18 & 2.08 & 0.119 & 0.85 \\
\hline 17 & & 0.14 & & 0.093 & 0.66 \\
\hline 18 & & 0.10 & & 0.066 & 0.47 \\
\hline 19 & & 0.06 & & 0.040 & 0.28 \\
\hline 20 & 0.80 & 0.13 & 3.16 & 0.131 & 0.83 \\
\hline 21 & & 0.10 & & 0.100 & 0.64 \\
\hline 22 & & 0.07 & & 0.070 & 0.45 \\
\hline 23 & & 0.04 & & 0.040 & 0.26 \\
\hline 24 & 0.60 & 0.08 & 5.59 & 0.125 & 0.78 \\
\hline 25 & & 0.06 & & 0.107 & 0.67 \\
\hline 26 & & 0.04 & & 0.071 & 0.45 \\
\hline
\end{tabular}

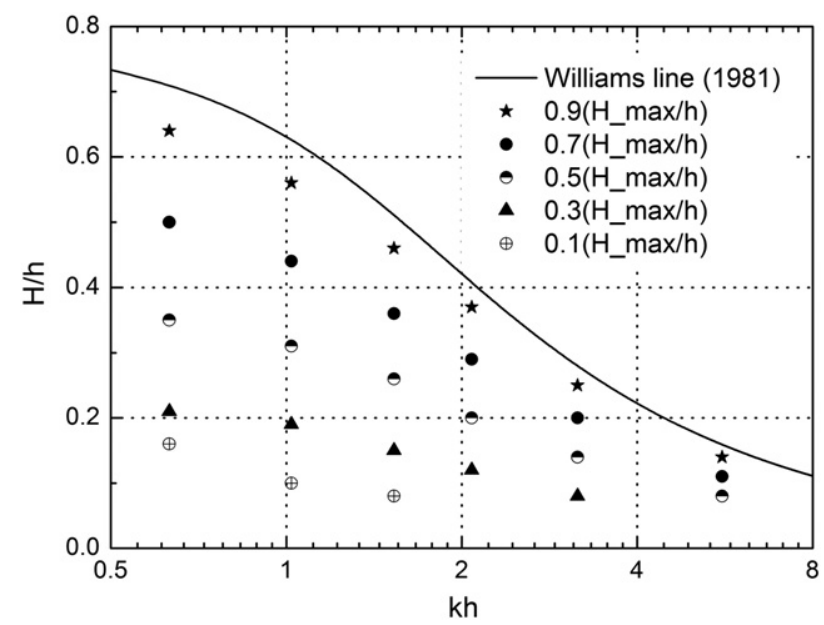

Fig. 16. Region of cases in Table 1, compared with Williams line (1981) which is the limit of the highest steady wave in practice.

gives the steepest stable wave. Considering the limitations of the wavemaker, no cases are possible with heights of less than $0.04 \mathrm{~m}$. Fig. 16 shows the distribution of the test cases in terms of the relative wave height versus the dimensionless water depth.

\subsection{Shallow water cases}

First of all, we consider a shallowest water case for a period of $T=$ $2.4 \mathrm{~s}$ and a height of $H=0.18 \mathrm{~m}$ with $k h=0.63$ and $H / L_{0}=0.022$. This case corresponds to a theoretical value of $S=0.94$, i.e., approximate to the limit of the validity of the theory as given by Eq. (41). Figs. 17 and 18 show time series of surface elevation at three different wave gauges using the first-order and second-order coupling signals, respectively. Both the first- and second-order coupling signal give quite good agreement at each position. To further investigate the efficiency of model when the nonlinearity parameters go out of the validity criterion, we therefore turn up the wave height to $H=0.32 \mathrm{~m}$ keeping the same period $T=2.4 \mathrm{~s}$, in which the nonlinearity parameter $S$ exceeds the limit considerably at $S=3.13$. The comparisons of surface profiles measured at three wave gauges with the theoretical results are shown in Figs. 19 and 20. Both the first- and second-order wave profiles agree fairly well with the theoretical solution at gauge 1 , but further down the flume (at gauges 2 and 3 ) this agreement is misleading because some unwanted spurious free wave appears in the wave troughs. These plots demonstrate that nonlinear effects have a tendency to require some time (or distance) to reveal their presence. The first-order

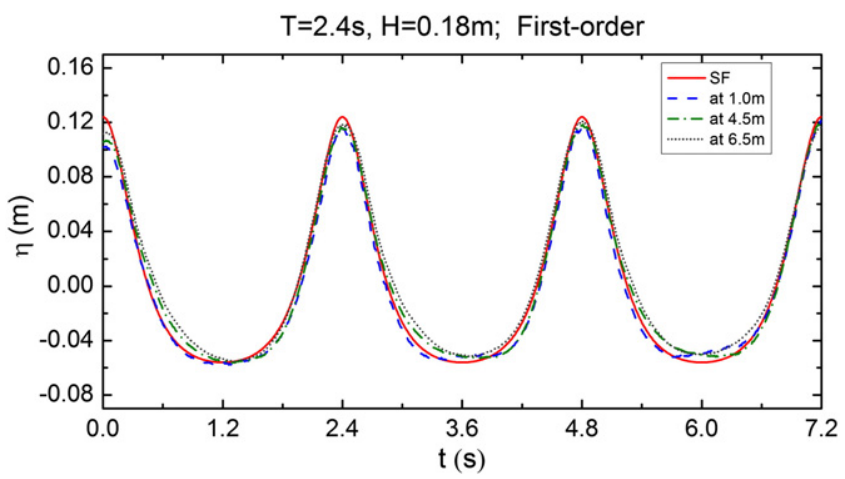

Fig. 17. Time series of surface elevations measured at different gauges in the flume using the first-order coupling signal for $T=2.4 \mathrm{~s}, H=0.18 \mathrm{~m}$, compared with the theoretical stream function theory (SF). 


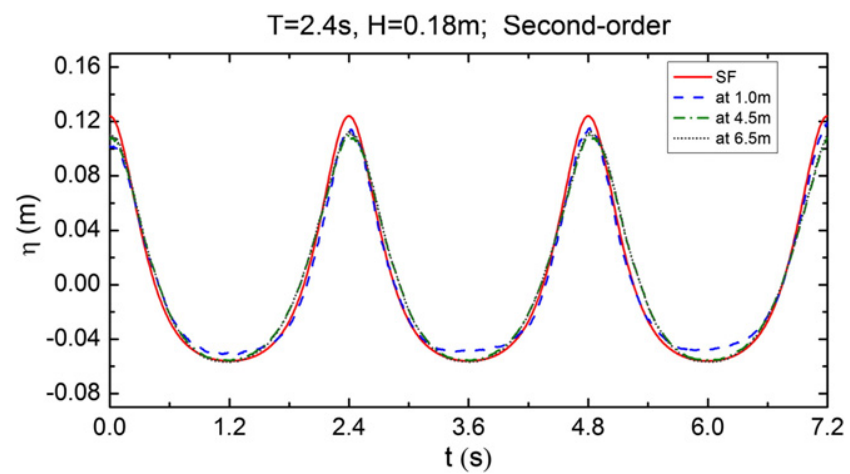

Fig. 18. Same as for Fig. 17, but using the second-order coupling signal.

coupling model appears to be unable to eliminate the spurious modes in this extreme case. However, a slight improvement can be observed in the second-order coupling model.

To quantify the accuracy and efficiency of the coupling model discussed in relatively shallow water, we consider the observed departure between experiments and theoretical solution for the selected two wave period of $T=2.4 \mathrm{~s}$ and $1.6 \mathrm{~s}$ in Table 1 using two statistical errors, i.e.

$r m s=\frac{1}{H_{T}} \sqrt{\frac{1}{T_{n}} \int_{0}^{T_{n}}\left[\eta_{T}(t)-\eta_{P}(t)\right]^{2} d t}$

$\alpha=\rho_{T P}\left(\tau_{0}\right) \frac{\sigma_{P}}{\sigma_{T}}=\frac{R_{T P}\left(\tau_{0}\right)}{\sigma_{T}^{2}}$

where $H_{T}$ is the theoretical wave height, $T_{n}$ the statistical time window. Subscripts " $T$ " and " $P$ " represent the time series in the theoretical and experimental result, respectively. $\sigma, \rho\left(\tau_{0}\right)$ and $R\left(\tau_{0}\right)$ are the standard deviation, standard covariance and the correlation coefficient function of two time series, respectively. rms (root-mean-square errors) is chosen to indicate the average deviation error of two wave trains. $\alpha$ varies between 0 and 1 with $\tau_{0}=0$ representing the non-dimensional correlation function of two time series. A perfect agreement between theoretical solution and experiment should result in $r m s \rightarrow 0$ and $\alpha \rightarrow 1$.

Fig. 21 gives the errors $r m s$ and $\alpha$ obtained by Eqs. (45-a) and (45-b) in the relatively shallow water wave cases, $T=2.4 \mathrm{~s}$ and

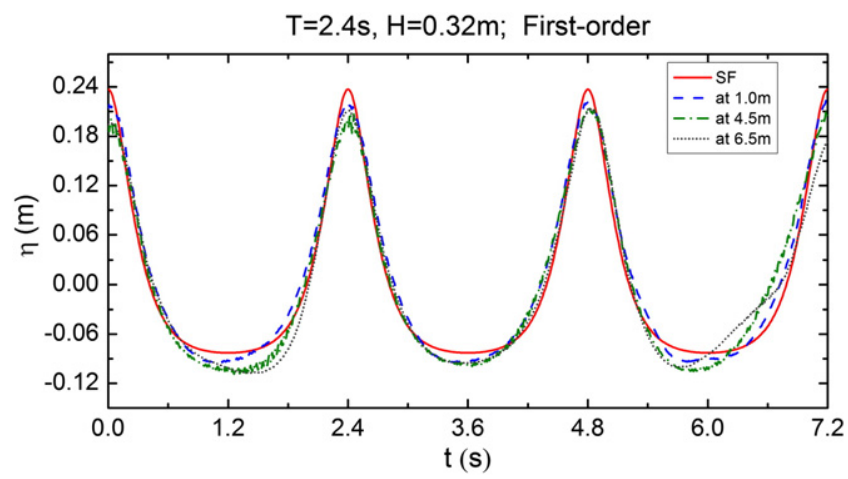

Fig. 19. Time series of surface elevations measured at different gauges in the flume using the first-order coupling signal for $T=2.4 \mathrm{~s}, H=0.32 \mathrm{~m}$, compared with the theoretical stream function theory (SF).

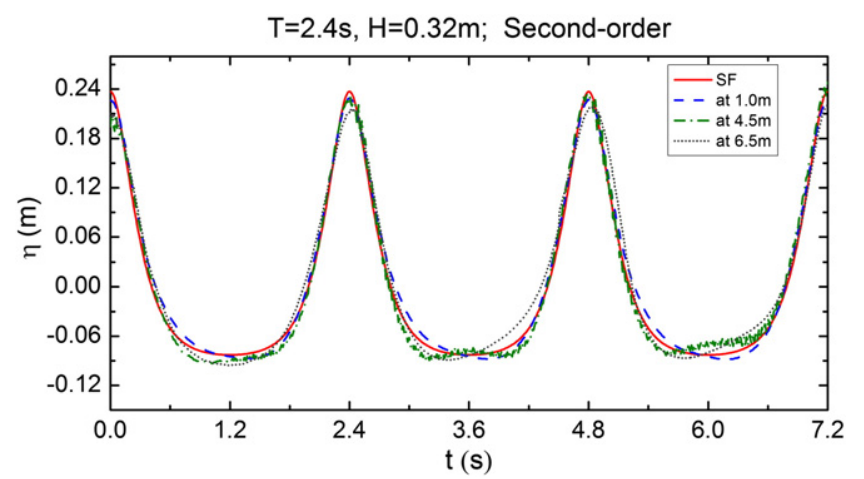

Fig. 20. Same as for Fig. 19, but using the second-order coupling signal.

$1.6 \mathrm{~s}$, at the three wave gauges for each wave height. This plot illustrates that the errors increase as the wave height increases. When the wave height is relatively small, both the first- and second-order coupling signals give small errors. The difference between the two coupling theories is not very clear for shallow water and weakly nonlinear waves, such as $H=0.08 \mathrm{~m}, 0.11 \mathrm{~m}, 0.25 \mathrm{~m}$ for $T=2.4 \mathrm{~s}$ and $H=0.05 \mathrm{~m}, 0.09 \mathrm{~m}$ for $T=1.6 \mathrm{~s}$. As the wave height grows, the results of the first-order coupling signal deteriorate. The second-order coupling signal, however, gives better results with almost all error rms being less than or around 5\%, and $\alpha$ being greater than 0.75 .

\subsection{Moderate and deep water cases}

We now turn to the cases of moderate and deep relative water depth with a period of $T=0.6-1.2 \mathrm{~s}$ corresponding to dimensionless water depth of $k h=2.08-5.59$. Time series of wave surface elevations measured at three wave gauges compared with the theoretical solutions are shown in Fig. 22 for the 90\% steepness cases, which correspond to the strongest nonlinearity for each period. These plots are for the results of the second-order coupling model. All the nonlinearity parameters $S$ are chosen to be below 1 . As it might be expected, the measured surface elevations at different wave gauges are in satisfactory agreement with related theoretical results. The wave shape is steady, and little spurious free waves can be observed. The conclusion holds even in the very deep water case.

Finally, Fig. 23 quantitatively collects the errors $r m s$ and $\alpha$ for each wave height tested over the remaining periods. The trend is very similar to the shallow water cases. The errors generally increase with larger relative water depth and wave height. For example, when $T=1.2 \mathrm{~s}$, $1.0 \mathrm{~s}$, and $0.8 \mathrm{~s}$, using the second-order signal, almost all the errors rms are smaller than those using the first-order coupling signal, and they are less than or around 5\% for most of the cases at all three gauges, except for some strongly nonlinear wave cases such as $T=1.2 \mathrm{~s}, H=$ $0.23 \mathrm{~m}$ and $T=0.8 \mathrm{~s}, H=0.13 \mathrm{~m}$. This plot also makes it clear that the merits of the second-order coupling signal become increasingly pronounced with increasing water depth and wave nonlinearity compared to the first-order coupling. This is especially true for the extreme case, $T=0.6 \mathrm{~s}, H=0.08 \mathrm{~m}$, the errors are very large when using first-order coupling signal. In this case, the average linear $r m s$ at the three gauges is $24.2 \%$, and the average $\alpha$ is 0.58 while the second-order coupling signal gives smaller average errors of $r m s=9.2 \%$ and $\alpha=0.88$.

The reason for this improvement is that the dispersion correction for the paddle position in the second-order signal is based on a full second-order theory and that the evanescent modes are included in the coupling wavemaker signal. These two improvements allow the physical model to capture more accurately the high order wave information that exists in the theoretical stream function solution. In shallow water, as discussed in Section 4, the second-order coupling theory tends 

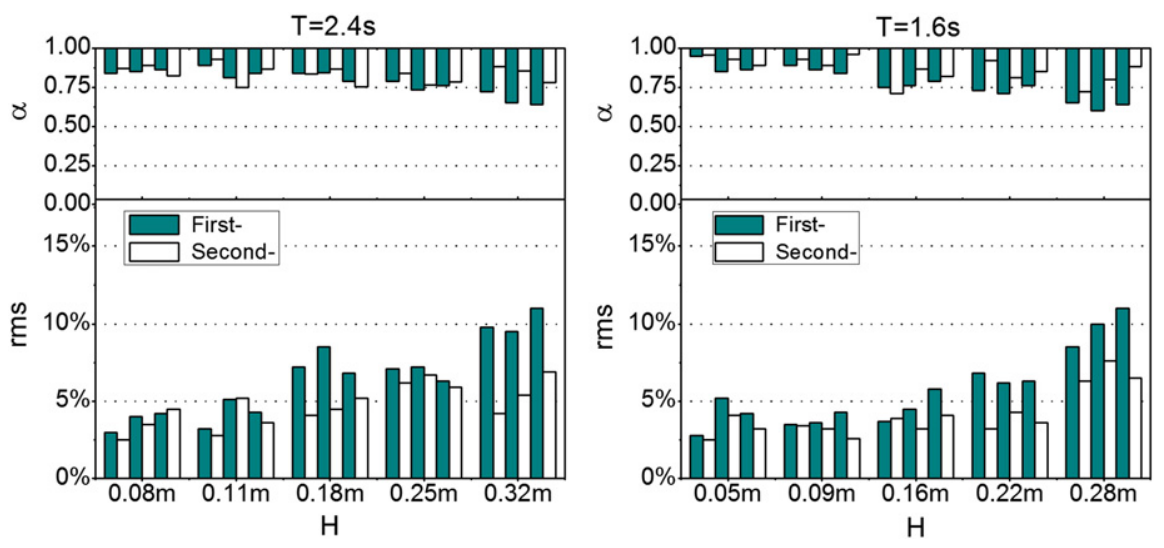

Fig. 21. The statistical errors $r m s$ and $\alpha$ for relatively shallow water wave cases.

to be consistent with the first-order theory since the second- and first-order coupling coefficients tend to be identical when the relative water depth tends to zero. However, as water depth and nonlinearity increases, the first-order dispersion correction based on linear theory becomes inadequate for correcting the high order component. The use of the second-order coupling theory helps to solve these problems.

\section{Conclusions}

The ad hoc unified wave generation theory of Zhang et al. (2007) has been extended to include a second-order correction term for the paddle motion based on wavemaker theory. The result provides a more accurate means of coupling numerical and physical wave tanks which includes both progressive and evanescent mode effects and covers both piston- and flap-type wavemakers.
A series of practical implementation problems have been solved to allow the theory to be applied in the laboratory. A theoretical analysis of the new formulation has been carried out based on the secondorder Stokes theory. Using a piston-type wavemaker, an experimental validation of the new model has also been conducted by considering a large range of cases based on stream function theory.

The new model collapses to the ad hoc wave generation theory of Zhang et al. (2007) and Yang et al. (2011b) in shallow water and to that of the approximate second-order coupling theory by Yang et al. (2011a) when the evanescent modes are neglected. Towards relatively deep water, the proposed second-order coupling theory provides an improved link between numerical and physical wave models and gives reduced spurious second-order free waves compared to existing methods. Work is currently underway to address the practical performance of the coupling model and to extend the present formulation to account for irregular and three-dimensional waves. a

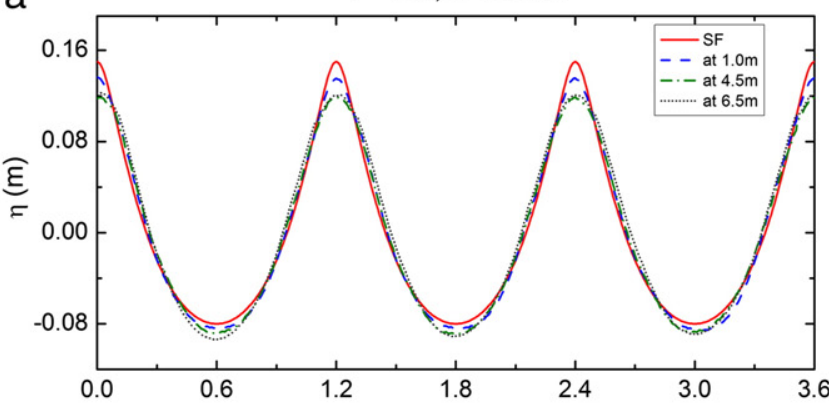

C

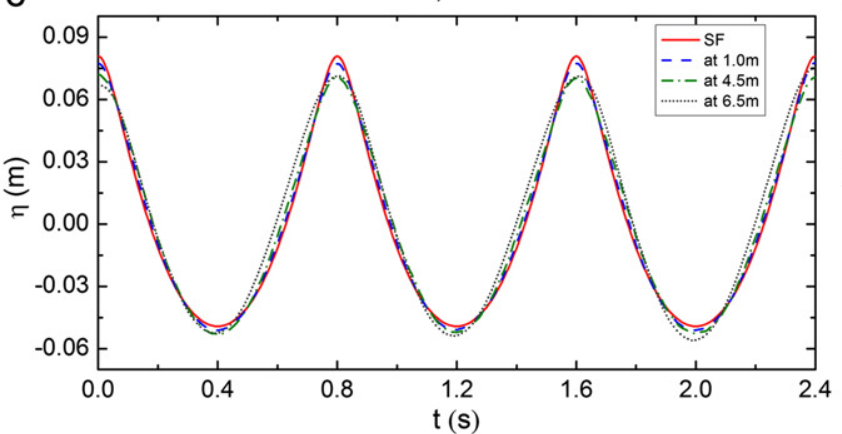

b

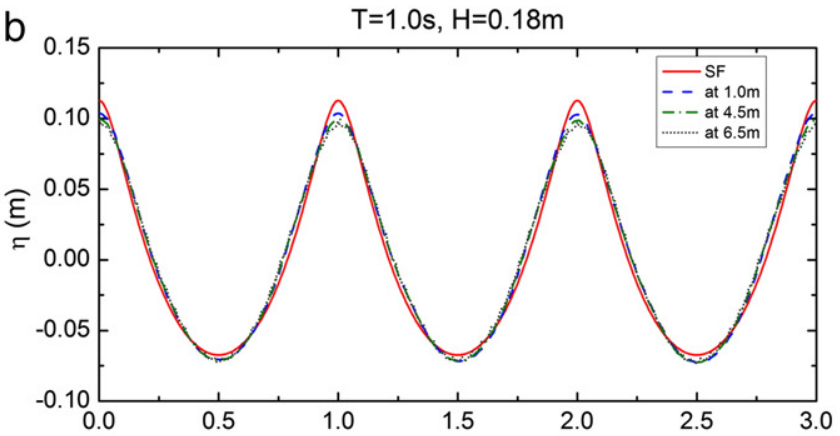

d

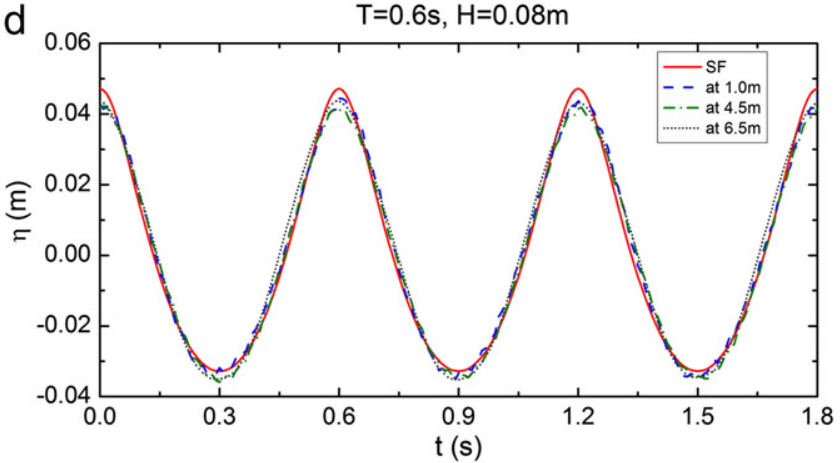

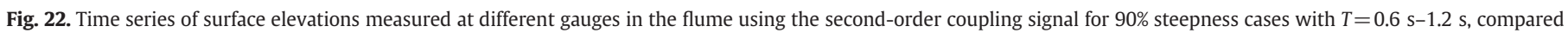
with the theoretical stream function theory (SF). 

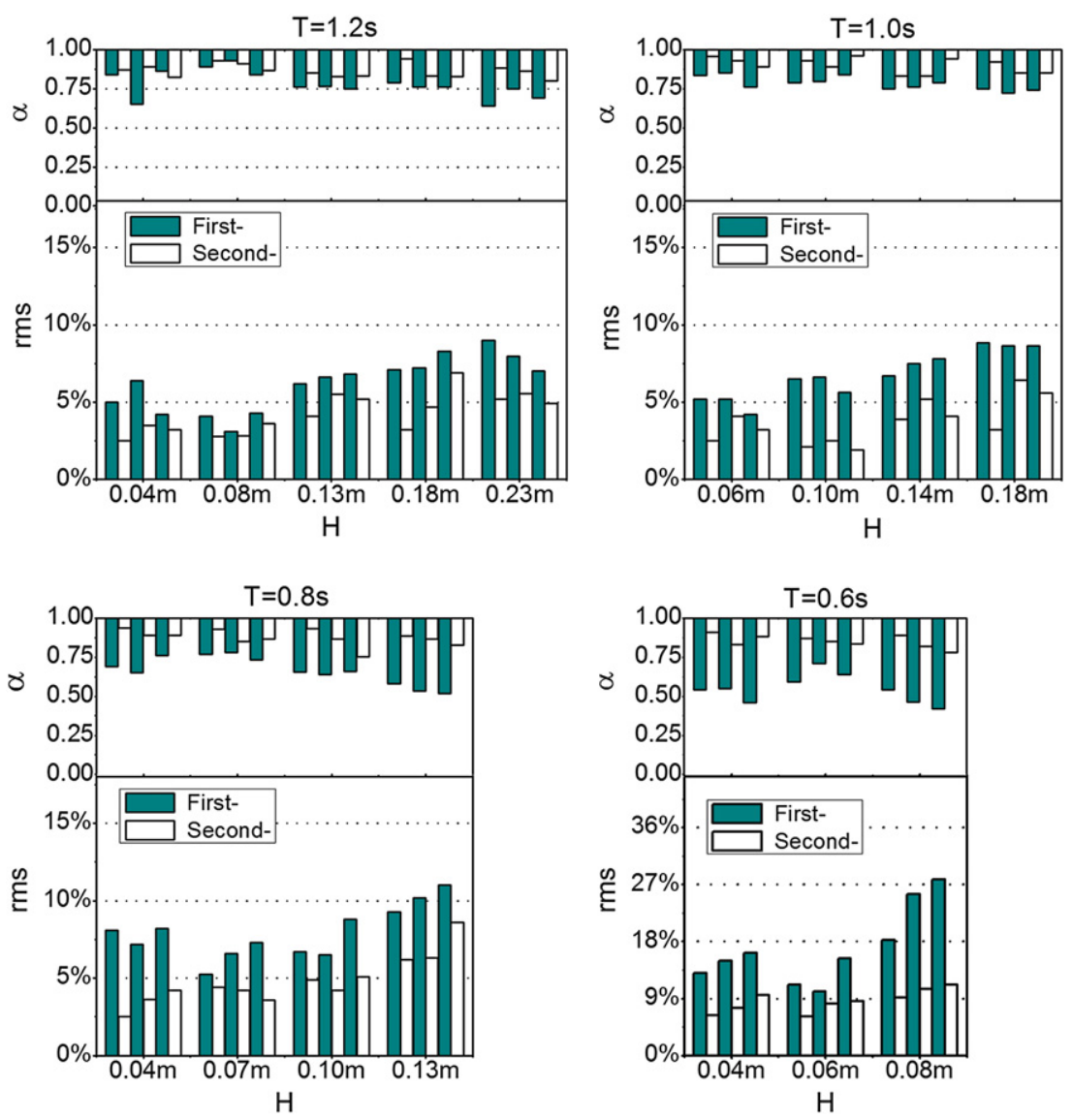

Fig. 23. The statistical errors rms and $\alpha$ for relatively deep water wave cases.

\section{Acknowledgments}

The corresponding authors would like to thank the China Scholarship Council (CSC) for financial support and scholarship award. Special thanks also to B. Mutlu Sumer, Bjarne Jensen and Martin Vistisen for the help during the physical model tests. We are grateful for the financial support by National Natural Science Foundation of China (grant no. 51079023, 50921001).

\section{Appendix A. Related coefficients used in coupling theory}

At first-order, the wave field is described by the velocity potential $\phi^{(1)}$. Herein, the transfer coefficients follow directly from the formulation in Madsen (1971) and are given by

$c_{j}=\frac{4 \sinh k_{j} h}{\left(2 k_{j} h+\sinh 2 k_{j} h\right)}\left(\sinh k_{j} h+\frac{1-\cosh k_{j} h}{k_{j}(h+l)}\right)$.

At second-order full theory, the expressions for $H_{j l}^{+}, D_{j l}^{+}, C_{p}^{(22)+}$ and $c_{p}^{(23)+}$ need to be defined. They are cited directly from Schäffer (1996) but only consider the special case of regular waves and the generation of superharmonics:

$H_{j l}^{+}=3 \omega^{3}-\frac{2 g^{2} k_{j} k_{l}}{\omega}-\frac{g^{2}}{2 \omega}\left(k_{j}^{2}+k_{l}^{2}\right)$

$D_{j l}^{+}=g\left(k_{j}+k_{l}\right) \tanh \left(k_{j}+k_{l}\right) h-4 \omega^{2}$

$(\mathrm{A}-3)$

$$
\begin{aligned}
c_{p}^{(22)+}= & \frac{h \cosh ^{2} K_{p}^{+} h}{2 g c_{0}^{2} \delta\left(K_{p}^{+}\right)}\left\{\sum_{j=0}^{\infty} \frac{c_{j} k_{j}^{2}\left(M_{2}\left(k_{j}, K_{p}^{+}\right)-3 \omega^{2}\right)}{k_{j}^{2}-\left(K_{p}^{+}\right)^{2}}\right. \\
& +\sum_{l=0}^{\infty} \frac{c_{l} k_{l}^{2}\left(M_{2}\left(k_{l}, K_{p}^{+}\right)-3 \omega^{2}\right)}{k_{l}^{2}-\left(K_{p}^{+}\right)^{2}} \\
& \left.-\frac{2 \omega}{g} \sum_{j=0}^{\infty} \sum_{l=0}^{\infty} \frac{c_{j} c_{l} H_{j l}^{+}\left(k_{j}+k_{l}\right)}{\left(k_{j}+k_{l}\right)^{2}-\left(K_{p}^{+}\right)^{2}}\right\} \\
c_{p}^{(23)+}= & \frac{4 \sinh K_{p}^{+} h}{\left(2 K_{p}^{+} h+\sinh 2 K_{p}^{+} h\right)}\left(\sinh K_{p}^{+} h+\frac{1-\cosh K_{p}^{+} h}{K_{p}^{+}(h+l)}\right)
\end{aligned}
$$

in which, $c_{p}^{(23)+}$ is the second-order transfer function, $M_{2}\left(\kappa, K_{p}^{+}\right)$and $\delta\left(K_{p}^{+}\right)$are given by

$$
\begin{aligned}
M_{2}\left(\kappa, K_{p}^{+}\right)= & -\frac{g}{(h+l)} \frac{2\left(K_{p}^{+}\right)^{2}}{\kappa^{2}-\left(K_{p}^{+}\right)^{2}}\left\{1-\frac{1}{\cosh \kappa h \cosh K_{p}^{+} h}\right. \\
& \left.-\frac{2 \omega^{4}\left(\kappa^{2}+\left(K_{p}^{+}\right)^{2}\right)}{g^{2} \kappa^{2}\left(K_{p}^{+}\right)^{2}}\right\}
\end{aligned}
$$

$\delta\left(K_{p}^{+}\right)=\frac{1}{2}\left(K_{p}^{+} h+\sinh K_{p}^{+} h \cosh K_{p}^{+} h\right)$ 
where $K_{p}^{+}$is second-order wave number, obtained by

$(2 \omega)^{2}=g K_{p}^{+} \tanh K_{p}^{+} h$

which is the dispersion equation generalized to the second-order complex numbers. The transfer function $F^{+}$found by Schäffer (1996) is described as

$F^{+}=-\frac{c_{0}^{(22)+}}{c_{0}^{(23)+}}$

where the subscript " 0 " denotes the progressive-wave components $(p \rightarrow 0)$.

\section{References}

Dean, R.G., Dalrymple, R.A., 1991. Water Wave Mechanics for Engineering and Scientific World Scientific, Singapore, pp. 295-324.

Fenton, J.D., 1988. The numerical solution of steady water wave problems. Computers and Geosciences 14 (3), 357-368.

Fenton, J.D., 1990. Nonlinear wave theories. In: Le Mehaute, B., Hanes, D.M. (Eds.), The Sea, pp. 3-25.

Flick, R.E., Guza, R.T., 1980. Paddle generated waves in laboratory channels. Journal of Waterway, Port, Coastal, and Ocean Engineering 106 (WW1), 79-97.

Fontanet, P., 1961. Théorie de la génération de la home cylindrique parun batteur plan. La Huille Blanche 16, 3-31 ((part 1) and 174-196 (part 2)).

Hudspeth, R.T., Sulisz, W., 1991. Stokes drift in two-dimensional wave flumes. Journal of Fluid Mechanics 230, 209-229.

Madsen, O.S., 1971. On the generation of long waves. Journal of Geophysical Research $76,8672-8683$
Ottesen-Hansen, N.E., Sand, S.E., Lundgren, H., Sorensen, T., Gravesen, H., 1980. Correct reproduction of long group induced waves. Proceedings of the 17th Coastal Engineering Conference, Sydney, Australia, pp. 784-800.

Sand, S.E., 1982. Long wave problems in laboratory models. Journal of Waterway, Port, Coastal, and Ocean Engineering 108 (WW4), 492-503.

Sand, S.E., Donslund, B., 1985. Influence of the wave board type on bounded long waves. Journal of Hydraulic Research 23, 147-163.

Schäffer, H.A., 1996. Second-order wavemaker theory for irregular waves. Ocean Engineering 23, 47-88.

Sulisz, W., Hudspeth, R.T., 1993. Complete second-order solution for water waves generated in wave flumes. Journal of Fluids and Structures 7, 253-268.

Synolakis, C.E., 1990. Generation of long waves in laboratory. Journal of Waterway, Port, Coastal, and Ocean Engineering 116 (2), 252-266.

Williams, J.M., 1981. Limiting gravity waves in water of finite depth. Philosophical Transactions of the Royal Society of London A 302, 139-188.

Yang, Z.W., Liu, S.X., Chang, J., Li, J.X., Sun, Z.B., 2011a. On the approximate secondorder coupling theory of numerical and physical wave model in flumes. Proceedings of the 21th International Offshore and Polar Engineering Conference, Maui, Hawaii, USA, June 19-24, 2011.

Yang, Z.W., Liu, S.X., Li, J.X., Sun, Z.B., 2011b. Coupling of numerical and physical model for simulation of wave propagation. Journal of Hydrodynamics, Series A 26 (3), 296-306.

Zhang, H., 2005. A deterministic combination of numerical and physical models for coastal waves. Ph.D. thesis, Technical University of Denmark, pp. 33-68.

Zhang, H., Schäffer, H.A., 2004. Waves in numerical and physical wave flumes - a deterministic combination. Proceedings of the 29th International Conference on Coastal Engineering, Lisbon, Portugal, September 2004.

Zhang, H., Schäffer, H.A., 2005. Waves in numerical and physical wave basins - a deterministic combination. Proceedings of Waves 2005, Madrid, Spain, July 2005.

Zhang, H., Schäffer, H.A., 2007. Approximate stream function wavemaker theory for highly non-linear waves in wave flumes. Ocean Engineering 34, 1290-1302.

Zhang, H., Schäffer, H.A., Jakobsen, K.P., 2007. Deterministic combination of numerical and physical coastal wave models. Coastal Engineering 54, 171-186. 